\title{
Molecular pharmacodynamics of emixustat in protection against retinal degeneration
}

\author{
Jianye Zhang, ${ }^{1}$ Philip D. Kiser, ${ }^{1,2}$ Mohsen Badiee, ${ }^{3}$ Grazyna Palczewska, ${ }^{4}$ Zhiqian Dong, ${ }^{1,4}$ Marcin Golczak, ${ }^{1}$ Gregory P. Tochtrop, ${ }^{3}$ \\ and Krzysztof Palczewski ${ }^{1,4}$
}

'Department of Pharmacology, Cleveland Center for Membrane and Structural Biology, School of Medicine, Case Western Reserve University, Cleveland, Ohio, USA. ²Luis Stokes Cleveland VA Medical Center, Cleveland, Ohio, USA. 르epartment of Chemistry, Case Western Reserve University, Cleveland, Ohio, USA. ${ }^{4}$ Polgenix Inc., Cleveland, Ohio, USA.

Emixustat is a visual cycle modulator that has entered clinical trials as a treatment for age-related macular degeneration (AMD). This molecule has been proposed to inhibit the visual cycle isomerase RPE65, thereby slowing regeneration of 11-cis-retinal and reducing production of retinaldehyde condensation byproducts that may be involved in AMD pathology. Previously, we reported that all-trans-retinal (atRAL) is directly cytotoxic and that certain primary amine compounds that transiently sequester atRAL via Schiff base formation ameliorate retinal degeneration. Here, we have shown that emixustat stereoselectively inhibits RPE65 by direct active site binding. However, we detected the presence of emixustat-atRAL Schiff base conjugates, indicating that emixustat also acts as a retinal scavenger, which may contribute to its therapeutic effects. Using agents that lack either RPE65 inhibitory activity or the capacity to sequester atRAL, we assessed the relative importance of these 2 modes of action in protection against retinal phototoxicity in mice. The atRAL sequestrant QEA-B001-NH2 conferred protection against phototoxicity without inhibiting RPE65, whereas an emixustat derivative incapable of atRAL sequestration was minimally protective, despite direct inhibition of RPE65. These data indicate that atRAL sequestration is an essential mechanism underlying the protective effects of emixustat and related compounds against retinal phototoxicity. Moreover, atRAL sequestration should be considered in the design of next-generation visual cycle modulators.

\section{Introduction}

Maintenance of photoreceptor light sensitivity and health critically depends on the provision of a constant supply of the visual chromophore 11-cis-retinal (1-4). This demand is met by a metabolic pathway known as the visual cycle that is composed of enzymes and retinoid-binding proteins expressed in the photoreceptors and the adjacent retinal pigment epithelium (RPE). An important first step in this series of reactions is the reduction of all-trans-retinal (atRAL) to all-trans-retinol (atROL), which serves to both clear the reactive retinaldehyde released from photoactivated visual pigments and enable the subsequent trans-cis isomerization step of the pathway (5). Certain environmental insults including prolonged exposure to intense light, in combination with an unfavorable genetic background, can overwhelm the retinal reductase capacity of the visual cycle, leading to pathological side reactions

Authorship note: Jianye Zhang and Philip D. Kiser contributed equally to this work. Conflict of interest: K. Palczewski and M. Golczak are co-inventors on US patent 8722669, "Compounds and methods of treating ocular disorders" and US patent 20080275134, "Methods for treatment of retinal degenerative disease," issued to Case Western Reserve University (CWRU). The values of these patents may be affected by this publication. CWRU may license this technology for commercial development. K. Palczewski is a member of the scientific board of Vision Medicine Inc., which is involved in developing visual cycle modulators. G. Palczewska is an employee of Polgenix Inc. K. Palczewski is the chief scientific officer (CSO) at Polgenix Inc. and an inventor on US patent 7706863, "Methods for assessing a physiological state of a mammalian retina," and US patent 8346345 B2, "Methods for assessing a physiological state of a mammalian retina," whose values may be affected by this publication. Submitted: January 13, 2015; Accepted: May 12, 2015.

Reference information: / Clin Invest. 2015;125(7):2781-2794. doi:10.1172/JCI80950. of retinaldehyde that ultimately disrupt retinal structure and function $(6,7)$. A clinical example is an inherited juvenile macular degeneration known as Stargardt disease, in which mutations in the photoreceptor-specific ATP-binding cassette transporter (ABCA4) result in delayed atRAL clearance $(8,9)$. The resulting increased concentrations of atRAL exert a direct cytotoxic effect on photoreceptors (10), in addition to contributing to the formation of side-products such as $N$-retinylidene- $N$-retinylethanolamine (A2E) and retinal dimer (11-13).

The focus on A2E as a primary toxin $(14,15)$, despite some evidence to the contrary $(16,17)$, led to the belief that inhibiting the visual cycle could have beneficial effects on averting light-induced retinal damage in certain forms of retinal and macular degeneration by lowering the density of visual pigments. This concept was first tested in patients treated with 13-cis-retinoic acid (isotretinoin) or its derivatives (18-20) and led to the development of an inhibitor of RPE65, the non-retinoid derivative of retinylamine (Ret- $\mathrm{NH}_{2}$ ) emixustat (previously known as ACU-4429) (21). Currently, emixustat hydrochloride is being tested in a phase IIb/III multicenter, randomized, double-masked, dose-ranging study by comparing its efficacy and safety with placebo for the treatment of dry age-related macular degeneration (AMD) (ClinicalTrials. gov identifier: NCT01802866) (21). Oral administration of this compound in humans results in dose-dependent suppression of the scotopic electroretinogram (ERG) b-wave, which is a proposed surrogate marker of its efficacy in treating dry AMD (21, 22). However, an extension of this presumed therapeutic effect is a debilitating inhibition of dark adaptation resulting in night blindness, an adverse effect also observed in patients treated with 
A

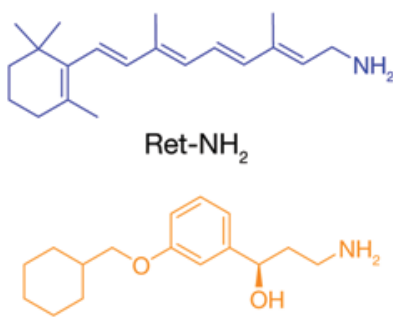

(R)-emixustat<smiles>NCCC(O)c1cccc(OCC2CCCCC2)c1</smiles>

(S)-emixustat

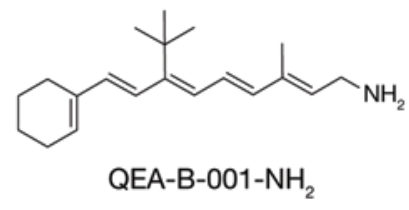

B

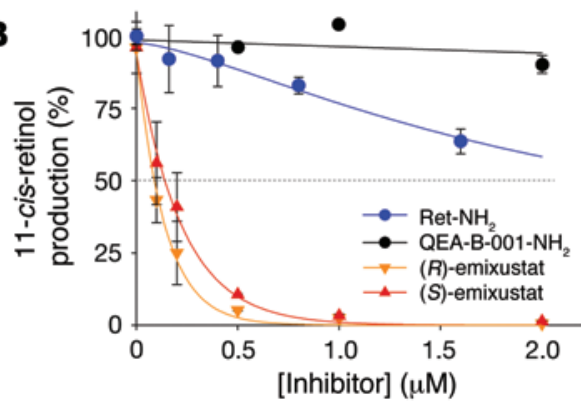

C

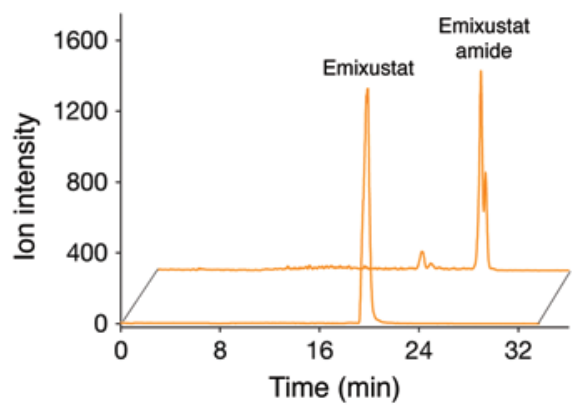

Figure 1. Inhibition of RPE65 by retinol analogs and formation of emixustat amide. (A) Primary amines used in this study that are structurally similar to retinol (vitamin A). (B) 11-cis-Retinol production in the presence of inhibitors depicted in A. Primary amines were preincubated with bovine RPE microsomes at room temperature for 5 minutes, then all-trans-retinol was added and the mixture incubated at $37^{\circ} \mathrm{C}$. All incubation mixtures were quenched by the addition of methanol after 1 hour of incubation. Inhibition of RPE65 enzymatic activity was measured as a decline in 11-cis-retinol production. Note that (S)-emixustat was 10 times more potent than Ret- $\mathrm{NH}_{2}$ in lowering 11-cis-retinol production. Without an inhibitor, typical activity was between 25 and $32 \mathrm{pmol} / \mathrm{minute}$, and this was set as $100 \%$ activity. (c) Extracted ion LC-MS chromatograms showing acylation of emixustat $(m / z=264.2$ $[\mathrm{MH}]^{+}$, lower trace) by LRAT in RPE microsomes to form corresponding emixustat palmitamide $\left(m / z=502.3[\mathrm{MH}]^{+}\right.$, upper trace). other inhibitors of the visual cycle (18-20). 11-cis-Retinal acts as an opsin inverse agonist (23). Therefore, suppression of 11-cis-retinal production by visual cycle inhibitors gives rise to unliganded, constitutively active opsins that initiate low-level activation of the phototransduction cascade (ref. 24 and references therein). Over time, this chronic signaling results in photoreceptor degeneration, as observed in patients with type 2 Leber congenital amaurosis (25), in Rpe65-KO mice (24), and in mice chronically treated with visual cycle inhibitors (26). Dyschromatopsia, attributed to aberrant rod effects on cone signaling pathways, was also frequently reported by patients treated with emixustat $(21,22)$. Despite lacking a retinoid backbone, emixustat structurally resembles retinol/ Ret- $\mathrm{NH}_{2}$, and thus its mechanism of action could be more complex than noted in the limited evaluations reported to date.

The photoreceptor atRAL concentration can reach $5 \mathrm{mM}$ if all rhodopsin is bleached $(4,27)$. In vitro, atRAL is cytotoxic, even at low micromolar concentrations (28-30). The atRAL released during photobleaching is primarily toxic to photoreceptor cells rather than to the RPE, as shown by 2-photon microscopy (TPM) (31). We demonstrated that the retina was spared from lightinduced degeneration by trapping the excess of this aldehyde with Ret- $\mathrm{NH}_{2}$ and non-retinoid primary amine-containing drugs (31, 32). This strategy makes use of aldehyde-selective chemistry to rapidly and reversibly conjugate atRAL with primary amine compounds that are structurally unrelated to Ret- $\mathrm{NH}_{2}$ (32). Notably, these compounds do not affect visual cycle enzyme function or the rate of rhodopsin regeneration in mice, indicating that atRAL sequestration constitutes their primary mode of action in prevention of retinal degeneration $(28,30,32)$. It has been demonstrated in mice lacking a functional visual cycle that the atRAL released from visual pigments present at baseline is sufficient to induce retinal phototoxicity (28). This result implies that ongoing bleaching and regeneration of visual pigments during light exposure enabled by the visual cycle is not necessary for the generation of light-induced retinal damage.
Complete elucidation of the molecular pharmacodynamics of emixustat is highly relevant to the continued development of visual cycle modulators as therapeutic agents for treatment of retinal disease. The structural similarity between Ret- $\mathrm{NH}_{2}$ and emixustat suggests that these agents could exert their protective effects through similar modes of action. In this study, we used mouse models to investigate the potency of RPE65 inhibition by emixustat, its utilization by lecithin:retinol acyltransferase (LRAT), the pharmacological selectivity of its 2 enantiomeric forms, its retention in the eye, and its ability to protect against acute light-induced retinal degeneration. Using a chemical biology approach informed by crystal structures of RPE65 in complex with emixustat, we assessed the relative importance of RPE65 inhibition and atRAL scavenging by visual cycle modulators in the protection against retinal phototoxicity in mice.

\section{Results}

Inhibitory effects of Ret- $\mathrm{NH}_{2}$ and emixustat in biochemical assays. Ret- $\mathrm{NH}_{2}$, the first described potent inhibitor of RPE65 (33), 2 enantiomers of emixustat, and QEA-B-001- $\mathrm{NH}_{2}$ (34) (Figure $1 \mathrm{~A}$ and supplemental material; available online with this article; doi:10.1172/JCI80950DS1) were tested for their ability to inhibit the RPE65-dependent retinoid isomerization reaction in a dose-dependent manner (Figure 1B). Amines were incubated with bovine RPE microsomes in the presence of atROL and the 11-cisretinoid-binding protein CRALBP. The decrease in 11-cis-retinol production reflects inhibition of RPE65 by a tested amine. The compounds had the following $\mathrm{IC}_{50}$ values: Ret- $\mathrm{NH}_{2}, 2.03 \pm 0.39$ $\mu \mathrm{M}$; $(S)$-emixustat, $150 \pm 24 \mathrm{nM}$; and $(R)$-emixustat, $91 \pm 6 \mathrm{nM}$. QEA-B-001- $\mathrm{NH}_{2}$ failed to prevent 11-cis-retinol production significantly at tested concentrations as previously reported (Figure 1B) (34). We did not detect any racemization of emixustat in RPE membranes or any significant binding to CRALBP.

Retinoid-like primary amines are acylated by the enzymatic action of LRAT (35). The resulting fatty acid amides of Ret- $\mathrm{NH}_{2}$ 

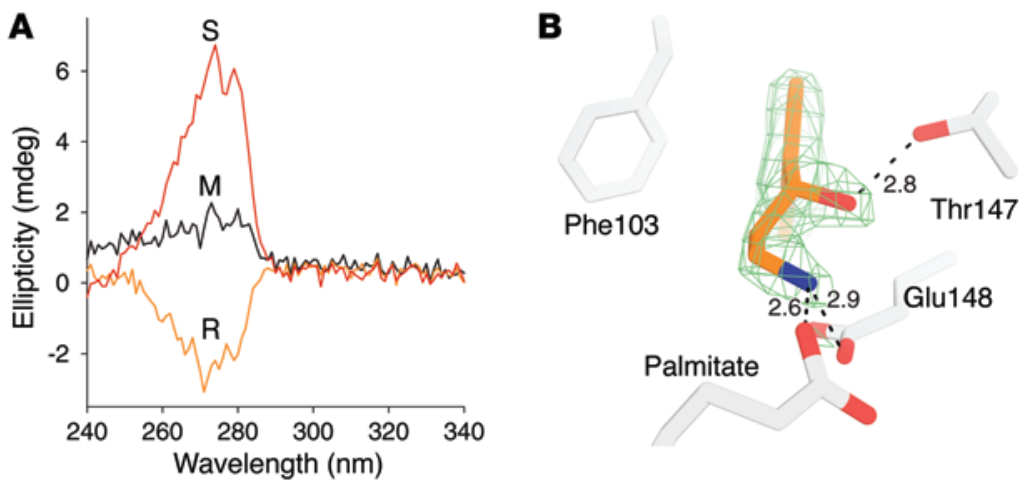

Figure 2. Analysis of emixustat enantiomer binding to RPE65. (A) Circular dichroism (CD) spectra of (R)-, (S)-, and racemic (M) emixustat. (B, C, and $\mathbf{D})$ Respective unbiased $\sigma A$-weighted omit Fo-DFc electron density maps at 3 root mean square deviation (RMSD) contour levels and associated models for the complexes obtained from racemic, $(R)$-, and $(S)$-emixustat. Dashed lines indicate hydrogen bonds with bond lengths given in $\AA$. For B, $(R)$-emixustat is clearly bound (compare with $\mathbf{C}$ ), despite the addition of equimolar concentrations of $(R)$ - and (S)-emixustat prior to crystallization.
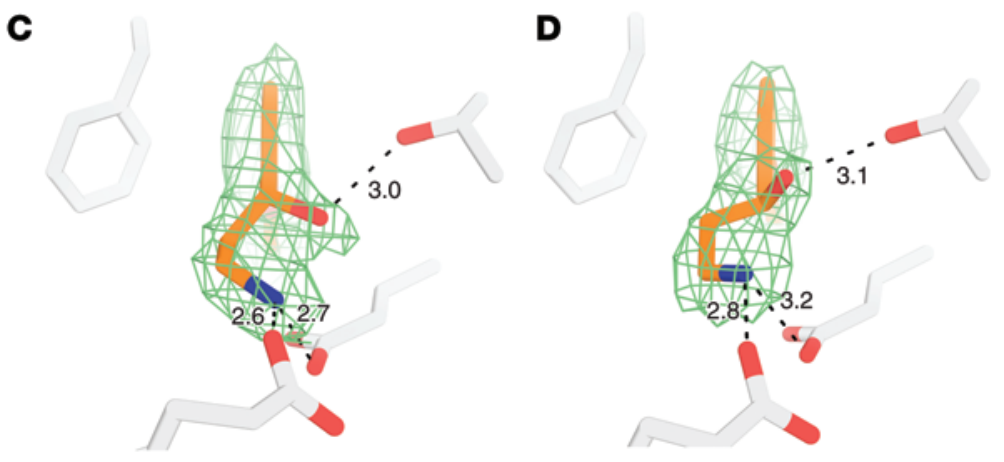

are storage forms of the compounds, which are then slowly hydrolyzed to evoke long-lasting suppression of retinoid isomerase activity (reviewed in refs. 36 and 37). Similar to Ret- $\mathrm{NH}_{2}$, emixustat was also efficiently amidated upon incubation with bovine RPE microsomes (Figure 1C), indicating that tissue uptake of the drug can be facilitated by LRAT enzymatic activity.

The structure of emixustat bound to RPE65. To gain insights into the molecular mode of RPE65 inhibition, we determined crystal structures of RPE65 in complex with emixustat. Structures were obtained in the presence of racemic emixustat as well as its pure enantiomers. We observed an unambiguous residual active site electron density corresponding to the bound inhibitor in all 3 cases (Supplemental Figure 1). Residual maps also indicated the presence of bound palmitate in the adjacent pocket of the active site, with its carboxylate oxygen forming a monodentate coordinate bond with the active site iron (Supplemental Figure 1). The hydroxyl moiety of emixustat was hydrogen bonded to the hydroxyl group of Thr147, whereas the primary amine was involved in polar interactions with the carboxylate groups of Glu148 and the bound palmitate ligand. Crystals obtained in the presence of racemic emixustat exhibited electron density consistent with an exclusive binding of the $(R)$-isomer, which consistent with the emixustat-bound RPE65 structure obtained in an alternative space group (38). The stereochemical assignment was further confirmed by structures obtained in the presence of pure $(R)$ - and $(S)$-emixustat, which revealed distinctly oriented electron densities for the hydroxyl moieties of these compounds (Figure 2). Density for the 3-amino-1-phenylpropan-1-ol moiety of (R)-emixustat was highly similar to that observed for the structure obtained from the racemic compound (Figure 2). These findings indicate that $(R)$-emixustat has higher binding affinity for RPE65 compared with that of the $(S)$-isomer, consistent with the slightly greater potency of the $(R)$-isomer compared with that of the $(S)$-isomer in the retinoid isomerase inhibition assay. The difference in binding affinity can be at least partially explained by the less favorable polar interaction we observed for the $(S)$-isomer compared with that for the $(R)$-isomer. Bond lengths for the hydroxyl-Thr147 O $\gamma$, amine-Glu148 Oع2, and amine-palmitate $\mathrm{O} 1$ interactions were $3.1 \AA$, $3.2 \AA$, and $2.8 \AA$ for the $(S)$-isomer compared with the $3 \AA$, $2.7 \AA$, and $2.6 \AA$ distances observed for the $(R)$-isomer, respectively. The difference in affinity was also evident in the disproportionately higher average $B$ factor for $(S)$-emixustat compared with that for $(R)$-emixustat, which could indicate either looser ligand binding or lower occupancy (Table 1).

In contrast to the polar end of the emixustat molecule, its cyclohexyl moiety exhibited structural flexibility, as evidenced by its higher atomic $B$ factor and its ability to adopt distinct conformations in different crystal forms (Supplemental Figure 2). In the structure obtained from racemic emixustat, the planes of the cyclohexyl and phenyl moieties were approximately perpendicular. This contrasts with the $(R)$ - and $(S)$-emixustat-bound structures, in which the cyclohexyl ring was rotated approximately $55^{\circ}$ clockwise when viewed along the cyclohexyl-methoxy bond. The conformation adopted in the racemic structure resulted in the presence of van der Waals and hydrophobic interactions between a lipophilic pocket formed by Ile259, Phe264, and Phe279 and the cyclohexyl moiety. In the enantiopure structures, the cyclohexyl moiety was rotated away from this lipophilic pocket toward a water-filled cavity. This conformational difference was accompanied by an inward movement of Phe264 and Phe196 toward the lipophilic cyclohexyl group, such that weak van der Waals contacts were established. In this conformation, the cyclohexyl group was also stabilized by van der Waals and hydrophobic interactions with the Thr147 C $\gamma$ and Ile259 C $\delta$ methyl groups. The conformational 


\section{Table 1. X-ray data and crystallographic refinement statistics}

\section{Data collection}

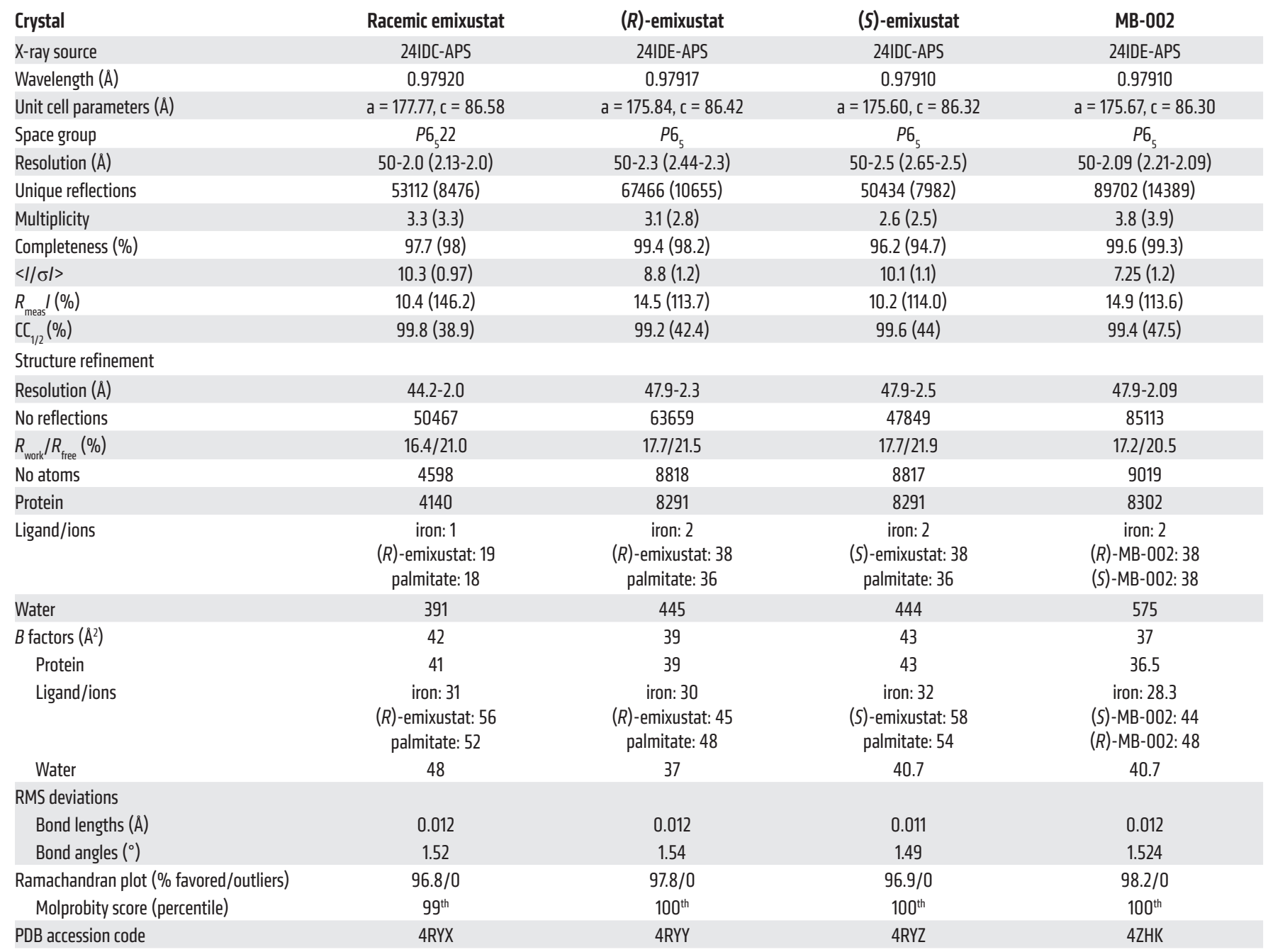

variability observed for this portion of the molecule probably relates to differences in mother liquor composition as well crystal packing. Conformational flexibility of the cyclohexyl moiety suggests that suitable modifications to this region of the molecule could lead to stronger interactions with surrounding protein residues and higher-affinity binding.

Inhibitory effects of emixustat on visual function. Stronger inhibition of 11-cis-retinol production by emixustat as compared with Ret- $\mathrm{NH}_{2}$ could lead to prolonged blockage of visual pigment regeneration in vivo. To test this hypothesis, we determined the rate of visual chromophore regeneration in WT mice treated with the drug and exposed to light. Because of the similar in vitro potency of the emixustat enantiomers, only the racemic mixture was investigated in vivo. Six hours after a $95 \%$ photobleaching, mice treated with $8 \mathrm{mg} / \mathrm{kg}$ emixustat recovered $35 \pm 1.7 \mathrm{pmol}$ 11-cis-retinal per eye, significantly less $(P<0.02)$ than did mice treated with the same amount of Ret- $\mathrm{NH}_{2}(118.2 \pm 27.7 \mathrm{pmol} /$ eye) (Figure 3A). At the same time point, 11-cis-retinal levels in untreated control animals was
$540 \pm 45 \mathrm{pmol} / \mathrm{eye}$. The difference between emixustat and Ret$\mathrm{NH}_{2}$ was significantly greater 20 hours after bleaching, when mice treated with Ret- $\mathrm{NH}_{2}$ had recovered approximately $68 \%$ of the visual chromophore, whereas animals treated by oral gavage with emixustat achieved only an approximate $12 \%$ recovery (Figure $3 \mathrm{~A}$ ). Additionally, the inhibitory effect of emixustat lasted longer; a single dose of $8 \mathrm{mg} / \mathrm{kg}$ caused nearly complete blockage of the visual cycle for over 7 days (Figure 3B). This effect could be attributed to a shifted equilibrium between the free and acylated form of emixustat. Unlike Ret- $\mathrm{NH}_{2}$, which can be detected predominantly in its acylated form (39), eye extracts from mice treated with emixustat revealed substantial amounts of free emixustat in addition to the corresponding amides (Figure 3C).

Protective effects of primary amines against light-induced retinal degeneration. To conduct a side-by-side evaluation of the protective effect of selected primary amines in an animal model for acute light-induced retinal degeneration, emixustat, Ret- $\mathrm{NH}_{2}$, or QEA-B-O01- $\mathrm{NH}_{2}$ was administrated to 4-week-old $\mathrm{Abca4} 4^{-/-}$ 

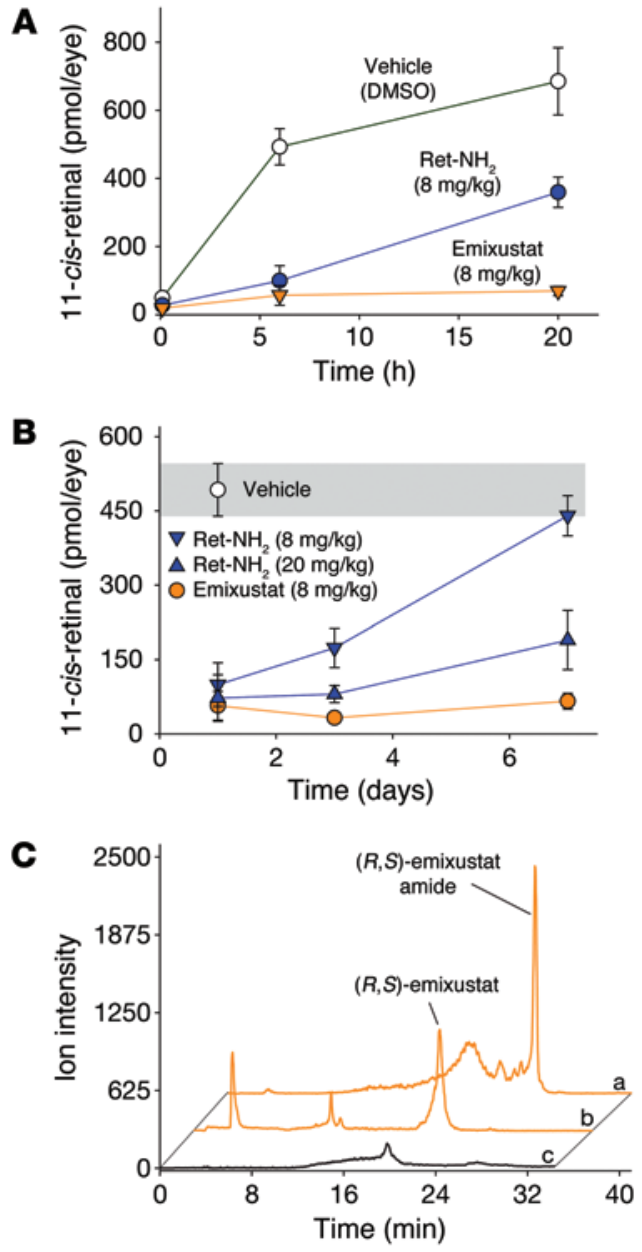

$\mathrm{Rdh}^{-/-}$mice. QEA-B-001- $\mathrm{NH}_{2}$ (Figure 1A), a primary amine incapable of inhibiting visual function (Figure 1B) and a substrate for LRAT, was used as a protective agent without strong inhibition of RPE65. Damaging retinal illumination (10,000 lux for 1 hour) was carried out 24 hours after drug administration. OCT images then were recorded after a 3-day dark adaptation period. In contrast to the 10-times higher potency of emixustat for inhibition of RPE65 in vitro, a lower dose of emixustat $(2 \mathrm{mg} / \mathrm{kg})$ did not completely protect the outer nuclear layer (ONL) in $\mathrm{Abca4}^{-/} \mathrm{Rdh}^{-/-}$mice, whereas $8 \mathrm{mg} / \mathrm{kg}$ of either emixustat or Ret- $\mathrm{NH}_{2}$ maintained both ONL thickness and light-scattering properties (Figure 4A). The average ONL thickness in mice receiving the $8 \mathrm{mg} / \mathrm{kg}$ dose was about $29 \pm 12 \mu \mathrm{m}$ for emixustat and $29 \pm 21 \mu \mathrm{m}$ for Ret- $\mathrm{NH}_{2}$ (Figure $4 \mathrm{~B}$ ), about $22 \pm 18 \mu \mathrm{m}(40)$ smaller than the ONL thickness of healthy mouse retina, indicating at least partial protection of photoreceptors. QEA-B-001- $\mathrm{NH}_{2}$ showed a protection at $80 \mathrm{mg} / \mathrm{kg}$, similar to that conferred by $2 \mathrm{mg} / \mathrm{kg}$ emixustat, with the ONL preserved as a diffuse light-scattering structure.

Impact of retinoid cycle modulator treatments on the quantity of enlarged photoreceptors. We had shown previously that photoreceptor cells are the primary sites for light-induced retinal degeneration in $\mathrm{Abca}^{-/-} \mathrm{Rdh}^{-/-}$mice and that the occurrence of enlarged, doughnut-shaped photoreceptors was the first observable indicator of ensuing photoreceptor demise (31). These changes in shape and fluorescence of photoreceptors preceded the formation of long
Figure 3. Inhibition of visual chromophore regeneration by emixustat and Ret- $\mathrm{NH}_{2}$ in C57BL6] mice. Recovery of 11-cis-retinal was measured in mouse eyes after exposure to bright light. (A) The progress of 11-cis-retinal recovery after light exposure in mice treated with either emixustat or Ret$\mathrm{NH}_{2}$. Both drugs were administrated at the same dose 24 hours prior to light exposure. (B) Persistence of the inhibitory effect in mice treated with Ret- $\mathrm{NH}_{2}$ or emixustat. Both drugs were administrated 1,3 , or 7 days before light exposure; the recovery of 11-cis-retinal was measured after a 6 -hours dark adaptation. (A and B) Mean values \pm SD are shown; $n=5-6$ for each data point. (C) Detection of emixustat and its amides in mouse eyes by LC-MS. The graph shows extracted ion chromatograms for $m / z=502.3$ $[\mathrm{MH}]^{+}$, representing emixustat palmitamide (a), and $m / z=264.3[\mathrm{MH}]^{+}$, corresponding to the free amine form of the compound (b). Chromatogram "c" reveals an intensity of $m / z=502.3$ ion for an eye extract obtained from an untreated animal.

wavelength-evoked $(850 \mathrm{~nm})$ fluorescent deposits in the RPE (3). To evaluate the protective impact of treatments with emixustat, Ret- $\mathrm{NH}_{2}$, and QEA-B-001- $\mathrm{NH}_{2}$, we pretreated these double-KO mice with these compounds before exposing them to bright light. On day 2 after such exposure, TPM was used to document the formation of enlarged photoreceptor outer segments in the retina (Figure 5). On average, we counted 43,242 enlarged photoreceptors per $\mathrm{mm}^{2}$ in the eyes of mice exposed to light and treated with vehicle only (soybean oil). Here, there were 438 enlarged photoreceptors per $\mathrm{mm}^{2}$ in mice treated with emixustat; 2,914/ $\mathrm{mm}^{2}$ in Ret- $\mathrm{NH}_{2}$-treated mice; $19,109 / \mathrm{mm}^{2}$ in QEA-B-001- $\mathrm{NH}_{2}-$ treated mice; and none in mice unexposed to light. The increase in fluorescence of the RPE in amine-treated mice is consistent with previous reports (35). Here, we found that the RPE fluorescence in emixustat-treated mice was $73.5 \pm 11$ gray values, and in the Ret- $\mathrm{NH}_{2}$-treated group, it was $118 \pm 6$ gray values. Taken together, these results indicate that photoreceptors were the targets for the protective action of the tested compounds.

Formation of retinylidene-emixustat Schiff base in vivo. Formation of a covalent bonded Schiff base (imine) is reversible under physiological conditions. Such formation requires 2 spatially arranged functional groups, a nucleophilic amino group and an electrophilic carbonyl group. For our selected compounds, the efficiency of imine formation depends on numerous factors including the chemical structures of the amine and aldehyde, solvent composition, $\mathrm{pH}$, and temperature. Examples of Schiff base formation in ocular tissue include transient formation of $\mathrm{N}$-retinylidene-phosphatidylethanolamine, a precursor of A2E in ROS of photoreceptors. Importantly, the propensity for Schiff base formation can be used to therapeutic advantage. Drugs that contain primary amines can react with an excess of free atRAL to form a reversible Schiff base, thus lowering the effective concentration of atRAL and preventing light-induced retinal degeneration (32). To verify whether emixustat, in addition to inhibiting RPE65, can potentially act as a scavenger for free atRAL, we investigated the formation and stability of its retinylidene Schiff base. As illustrated in Figure 6, A and B, this conjugation occurred readily under a broad range of primary amine/atRAL ratios. Thus, in contrast to Ret- $\mathrm{NH}_{2}$, the reaction equilibrium strongly favors efficient Schiff base formation between emixustat and atRAL.

To evaluate whether conjugates between atRAL and emixustat are formed in vivo, we examined the retinoid composition of eyes 


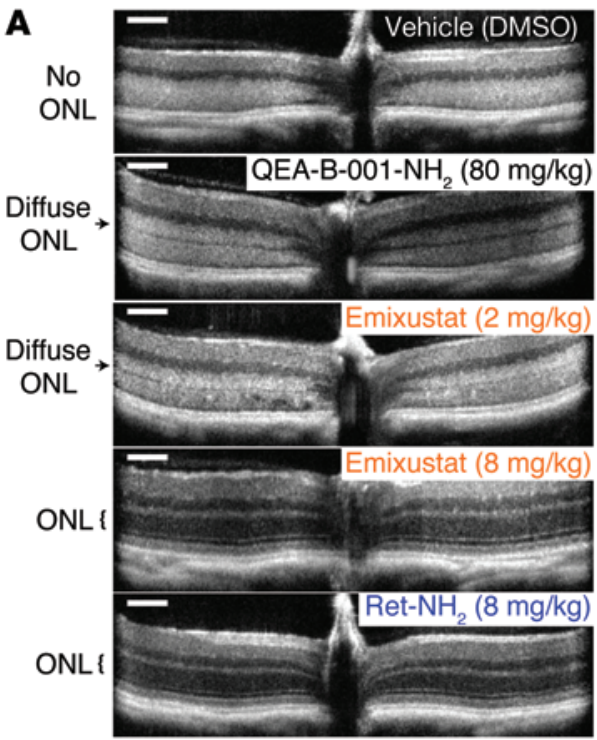

B

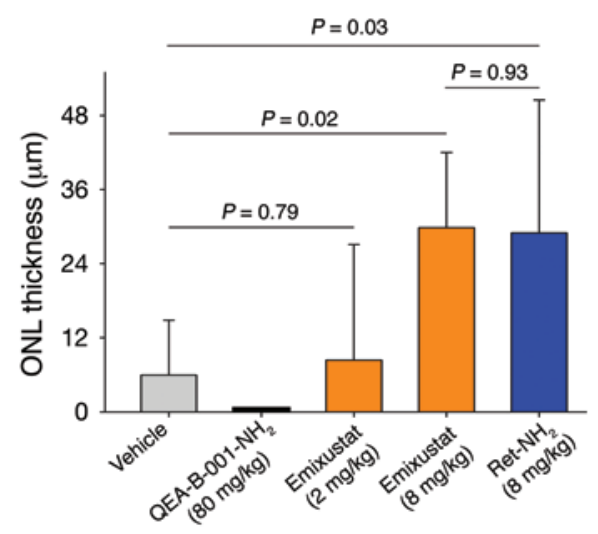

Figure 4. Protective effects of primary amines against light-induced retinal degeneration in $\mathbf{A b c a 4}^{-/-} \mathbf{R d h}^{-/-}$mice. Four-week-old $A b \mathrm{Ca4}^{-/-} \mathrm{Rdh}^{-/-}$mice treated with the tested amines were kept in the dark for 24 hours, bleached with 10,000 lux light for 1 hour, and then kept in the dark for 3 days. (A) Representative OCT images of mice treated with QEAB-001- $\mathrm{NH}_{2}$ ( $80 \mathrm{mg} / \mathrm{kg}$ ), emixustat $(2 \mathrm{mg} / \mathrm{kg}$ and $8 \mathrm{mg} / \mathrm{kg}$ ), and Ret- $\mathrm{NH}_{2}(8 \mathrm{mg} / \mathrm{kg})$. A dramatic decrease in the ONL indicates advanced retinal degeneration. Scale bars: $100 \mu \mathrm{m}$. (B) Quantification of the protective effects of QEA-B-001- $\mathrm{NH}_{2}$, emixustat, and Ret- $\mathrm{NH}_{2}$ are shown by measuring the average thickness of the ONL. Only non-scattering ONL layers were measured. For images in which the ONL layer boundary was obscured due to light scattering, the $\mathrm{ONL}$ length was recorded as $0 \mu \mathrm{m}$. Mean values \pm SD are shown; $n=5-6$ for each group $P$ values were calculated by ANOVA. from $\mathrm{Abca4}^{-/-} \mathrm{Rdh}^{-/-}$mice gavaged with $8 \mathrm{mg} / \mathrm{kg}$ of the amine 2 hours prior to light exposure (10,000 lux for 15 minutes). After organic extraction, retinoids and retinyl imines were separated by HPLC and identified on the basis of a comparison of their elution times with those of synthetic standards and their characteristic maximum UV/visible (UV/Vis) absorption at $450 \mathrm{~nm}$ upon protonation. Additionally, the molecular identity of retinylidene-emixustat was confirmed by tandem mass spectrometry (MS/MS) (Figure 6, $\mathrm{C}-\mathrm{F}$ ). These analyses clearly indicated that systemic administration of emixustat causes Schiff base formation with atRAL within mouse eye tissue. Notably, retinylidene-emixustat was detectable at very low doses, e.g., $8 \mathrm{mg} / \mathrm{kg}$ of the compound. Thus, the higher efficiency of Schiff base formation observed in vitro was confirmed by our in vivo findings. This observation indicates that emixustat can moderate atRAL concentrations upon photoactivation of rhodopsin and thus prevent photoreceptor degeneration.

Absence of the retinal protective effect of an emixustat derivative lacking the ability to sequester free retinal. To further discriminate between the atRAL sequestrant and RPE65 inhibitory mechanisms of action of emixustat in retinal protection, we sought to identify a compound specifically lacking the former activity. The amine moiety of emixustat is critical for its ability to react with atRAL to form an innocuous Schiff base conjugate. On the basis of the RPE65-emixustat crystal structures, we reasoned that substitution of the primary amine with a hydroxyl moiety could maintain high-affinity RPE65 binding, while eliminating atRAL scavenging activity (Figure 7A). This diol derivative, referred to as MB-002, was active as an RPE65 inhibitor both in vitro and in vivo (Figure 7, B and C and Supplemental material), with potencies comparable to that of Ret- $\mathrm{NH}_{2}$. The incomplete in vitro inhibition was likely due to its esterification by LRAT at higher concentrations. The crystal structure of RPE65 obtained in the presence of MB-002 revealed that this compound was indeed bound to the active site in a fashion similar to that of emixustat (Figure 8). Surprisingly, the electron density in the cavity occupied by palmitate in the emixustat-bound structures indicated the presence of a second active site-bound MB-002 molecule. MB-002 bound in this secondary site forms a direct interaction with the iron cofactor, a hydrogenbonding interaction with Tyr338, and a number of van der Waals contacts with non-polar side chains (Figure 8). The electron density was less well defined for the molecule at this secondary site, and binding at this position may have been enabled only by the high concentration of MB-002 (5 mM) used in the crystallization trials. Despite its strong and direct inhibition of retinoid isomerase activity, MB-002 failed to protect mice from light-induced retinal damage, as assessed by OCT and TPM (Figure 7, D and E). Moreover, to confirm that differences in the protective effects of emixustat versus MB-002 against light-induced retinal degeneration are not limited to mice lacking ABCA4 and $\mathrm{RDH} 8$, we compared the impact of these compounds on retina preservation in $\mathrm{BALB} / \mathrm{c}$ mice (41). Again, we found that treatment with emixustat fully protected the retina from light-induced damage, whereas the protection conferred by treatment with MB-002 was minimal (Figure 9). Even though in these mice we had to use double the intensity of light and double the duration of light exposure as compared with the exposure used in $\mathrm{Abca4^{-/- }} R d h 8^{-/-}$mice, there were fewer damaged photoreceptors than in $\mathrm{Abca4}^{\mathrm{I}^{--}} \mathrm{Rdh}^{-{ }^{--}}$mice. These experiments in mice with pharmacologically suppressed visual cycles demonstrate that light-induced retinal degeneration in mice is predominantly induced by atRAL that is released from visual pigments after the initial photobleaching rather than from visual pigments that were regenerated during the period of light exposure.

\section{Discussion}

The development of safe and effective small-molecule therapeutics for blinding retinal degenerative diseases still remains a major challenge (42). Several different approaches targeting specific enzymes or signaling pathways have yielded a number of promising leads, among which is modulation of visual cycle function $(43,44)$. Two experimentally validated methods for prevention of light-induced retinal degeneration involve (a) inhibition of the retinoid cycle $(7,30)$ and (b) sequestration of excess atRAL by drugs containing a primary amine group (32). Conceptually, these methods are derived from 2 different ideas but have a com- 

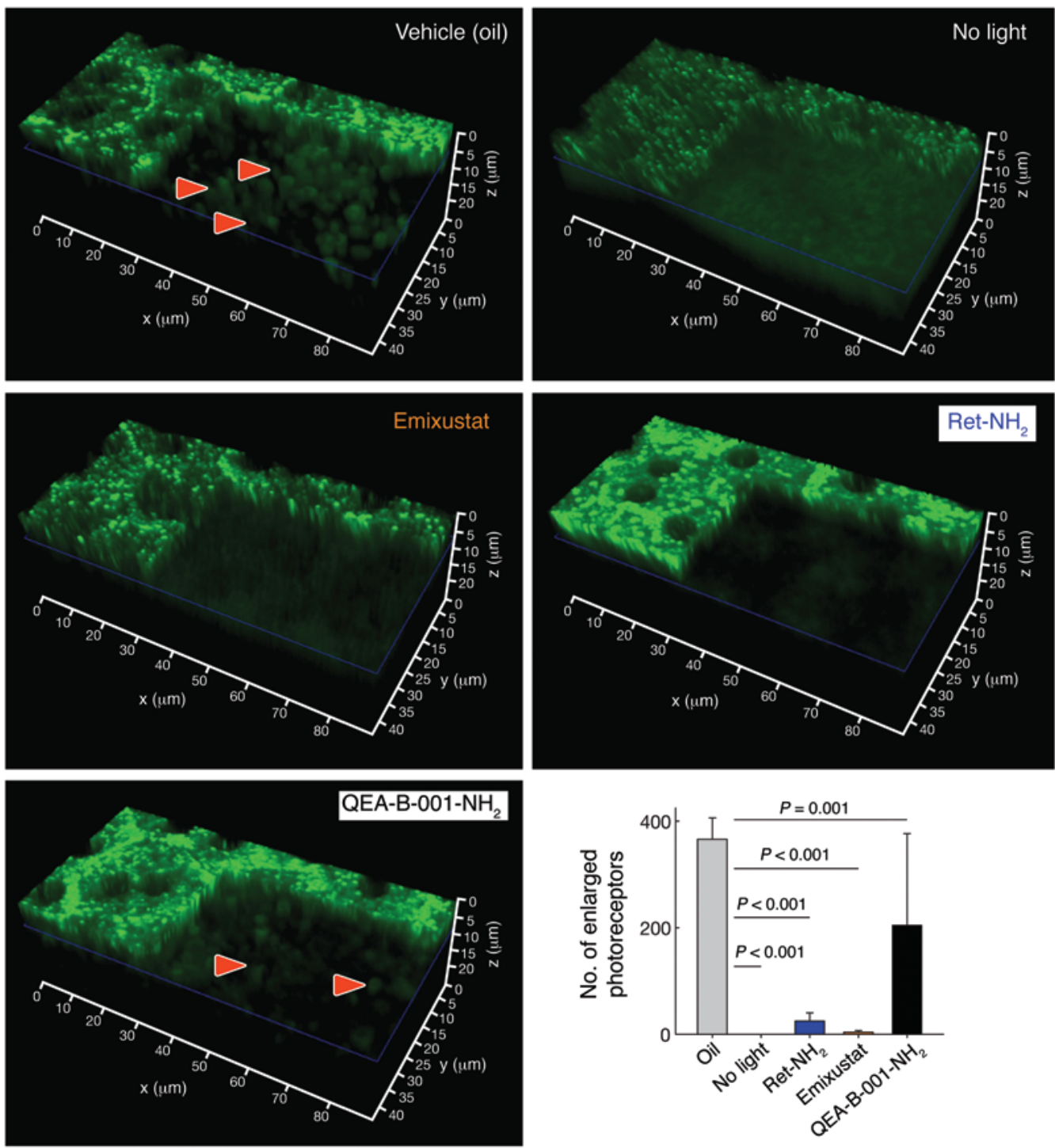

Figure 5. Impact of treatment with retinoid cycle modulators on the quantity of enlarged photoreceptors in $\mathrm{Abca4}^{-/-} \mathbf{R d h}^{-/-}$ mice. $A b \mathrm{ca}^{-/-} \mathrm{Rdh}^{-/-}$mice were exposed to bright light on the day after they were gavaged with oil (control), Ret- $\mathrm{NH}_{2}(80 \mathrm{mg} / \mathrm{kg}$ ), emixustat (racemic, $40 \mathrm{mg} / \mathrm{kg}$ ), or QEA-B-001- $\mathrm{NH}_{2}(80 \mathrm{mg} / \mathrm{kg}$ ). TPM images of intact double-KO mouse eyes were obtained on day 2 after exposure to light, and representative images for each group are shown. In each TPM 3D section, RPE cells are at the top of the section, and the photoreceptor cell layer section is shown $9-12 \mu \mathrm{m}$ below the RPE. Treatments are indicated in each image. Enlarged photoreceptor cells, indicated with red arrowheads, were noted only in animals that were exposed to light and treated with oil or QEA-B-001$\mathrm{NH}_{2}$. Enlarged photoreceptor cells were counted (graph) for animals in each treatment group; mean values are displayed. Error bars indicate SD; $(n=4)$; $P$ values were calculated by ANOVA and represent the statistical significance for each treated group versus control animals exposed to light and treated by gavage with oil. mon goal of reducing atRAL and/or atRAL condensation product toxicity as discussed below.

Blocking of the visual cycle would slow down rhodopsin regeneration through the retinoid cycle and, in turn, lower the production of toxic atRAL and its conjugation product A2E. For this mode of action, a low concentration of a potent inhibitor would be most desirable. An example of such an RPE65 inhibitor derived from Ret- $\mathrm{NH}_{2}$ is emixustat, which is currently undergoing clinical trials $(21,22)$. Comparison of these 2 primary amines indicated a 15 -fold greater inhibitory potency of emixustat in our standard retinoid isomerization assay (Figure 1B). However, marked suppression of the visual cycle is predicted to have highly undesirable consequences. Prolonged inhibition of 11-cis retinoid production causes accumulation of unliganded opsin, a condition that resembles Leber congenital amaurosis (LCA) or vitamin A deprivation, and this gives rise to retinal dystrophies as demonstrated in mouse models $(24,26)$. In particularly, cone photoreceptor cells, key cells for supporting human vision in a modern world bathed in artificial illumination, are perilous to chromophore deprivation $(26,45-48)$. Thus, precise dosing is critical for drugs with potent detrimental RPE65 inhibitory properties and possessing protective sequestration activity.

The crystal structures of RPE65 in complex with emixustat provide a molecular basis for understanding its strong inhibitory activity. Emixustat interacts with the retinoid-binding site of RPE65 and thus can be structurally classified as a competitive inhibitor. The hydroxyl group of the emixustat chiral center is appropriately positioned to participate in a hydrogen-bonding interaction with Thr147, an interaction that is not possible for Ret$\mathrm{NH}_{2}$. The importance of this polar interaction is evident from the preferential binding of $(R)$-emixustat in the presence of an equimolar concentration of $(S)$-emixustat as well as from the more chemically favorable and better-ordered binding of $(R)$-emixustat compared with that of $(S)$-emixustat in structures obtained from enantiopure compounds. Appropriate positioning of the primary amine group in emixustat allows ion-pairing interactions with Glu148 and the bound palmitate carboxylate groups. Because Ret- $\mathrm{NH}_{2}$ is extended by 1 methylene group compared with emixustat, it is conceivable that this ion-pairing interaction may be less favorable for Ret- $\mathrm{NH}_{2}$. The 2 different crystal forms reported here 
A

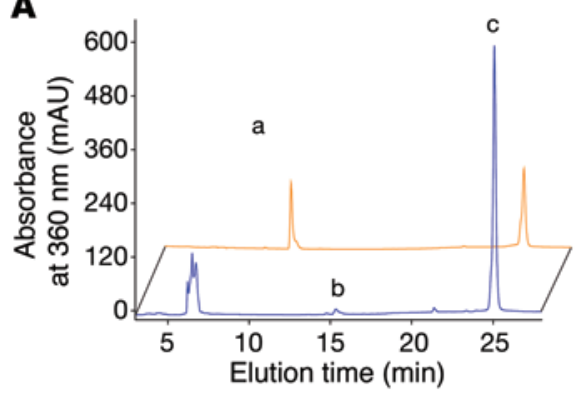

C

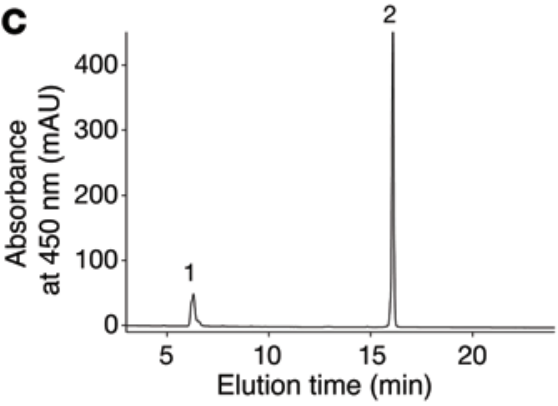

E

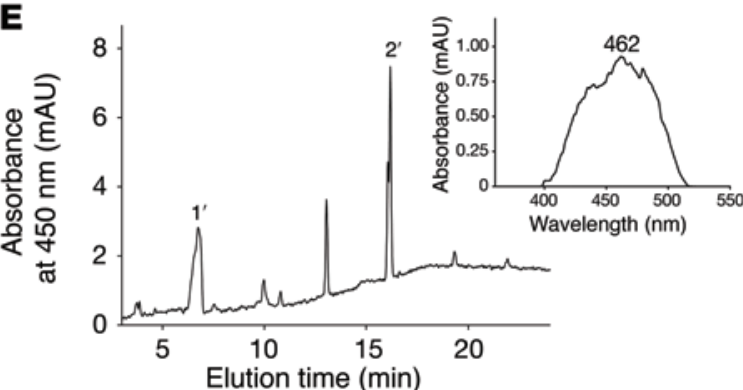

B

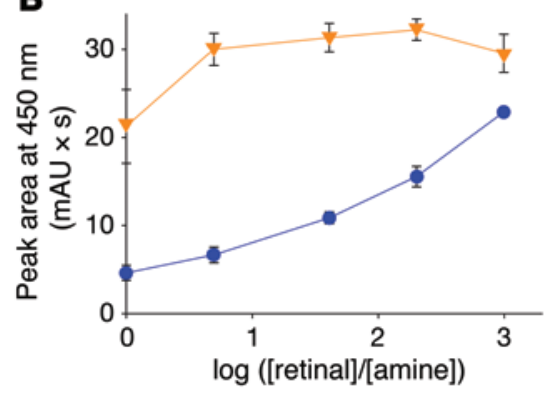

D

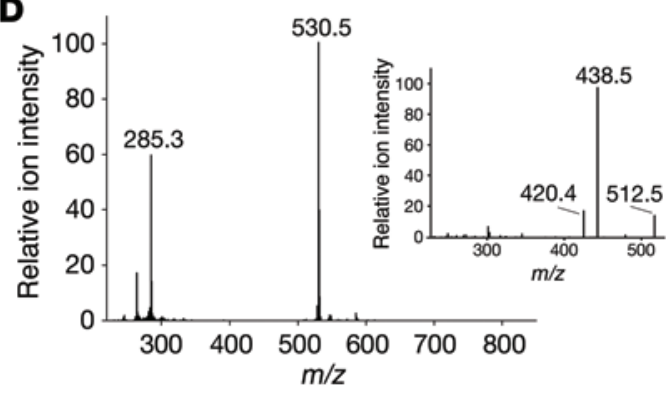

F

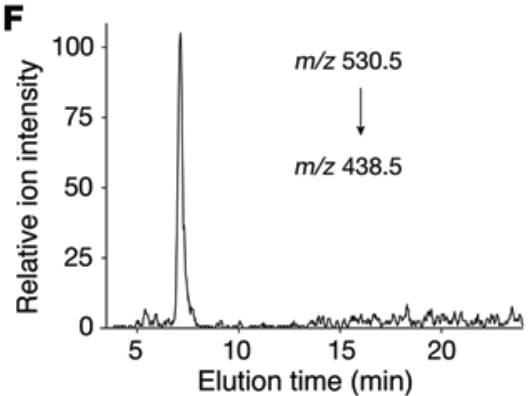

Figure 6. Emixustat-atRAL Schiff base formation in vitro and in vivo. (A) Chromatographic separation and detection of primary amines, atRAL, and their Schiff base conjugates. Emixustat or Ret- $\mathrm{NH}_{2}$ was incubated with atRAL for 2 hours, and the mixture was separated on a C-18 column. Representative HPLC chromatograms indicate the formation of an emixustat Schiff base (peak "a") and Ret- $\mathrm{NH}_{2}$ Schiff base (peak "b") at an aldehyde/amine ratio of 1:1. Peak " $c$ " corresponds to unreacted atRAL. (B) Quantification of Schiff bases formed upon incubation of increasing concentrations of atRAL with either $0.2 \mathrm{mM}$ emixustat (inverted triangles) or Ret- $\mathrm{NH}_{2}$ (circles). (C) The chromatogram illustrates separation of an emixustat Schiff base standard. Peak 1 , between 6 and 7 minutes, corresponds to the emixustat retinylidene Schiff base as indicated by its MS spectrum (D) with a dominant ion of $m / z=530.5$ corresponding to $[\mathrm{MH}]^{+}$of the emixustat-retinylidene Schiff base. Peak 2 corresponds to atRAL. Inset in $\mathbf{D}$ shows the MS/MS fragmentation pattern of the $\mathrm{m} / \mathrm{z}=530.5$ parent ion. (E) Detection of emixustat-retinylidene Schiff base in vivo; $8 \mathrm{mg} / \mathrm{kg}$ emixustat was administered to $\mathrm{Abca}^{-/-}$Rdh $8^{-/-}$mice 2 hours before light bleaching. After bleaching, mice were euthanized, and eye extracts were analyzed by LC-MS. Peak 1 ' observed in the chromatogram corresponds to an emixustat Schiff base. Its identity was confirmed by its UV/Vis spectrum with a characteristic absorbance maximum of $462 \mathrm{~nm}$ (inset) and by MS (F), in which fragmentation of the $m / z=530.5$ ion yielded the product ion at $m / z=438.5$, identical to that shown in $\mathbf{D}$. $m A U$, milliabsorbance units.

demonstrate that the cyclohexyl moiety of emixustat has a significant degree of conformational flexibility within the RPE65 active site. Many of the residues in close proximity to this portion of the molecule belong to a mobile region of RPE65 that also serves to anchor the protein to microsomal membranes. Thus, it is likely that conformational differences in these regions alter the shape of the cyclohexyl-binding site, which in turn gives rise to the 2 distinct conformations observed in the crystal structures. Should it be desirable to enhance the emixustat binding affinity for RPE65, it would appear that the methylcyclohexyl moiety would be a strong candidate site for chemical modification.

The second strategy involves sequestration of atRAL. Rod photoreceptor cells express huge amounts of rhodopsin estimated at a level of 3 to $5 \mathrm{mM}$ (4). Thus, a $10 \%$ bleaching will liberate $300-500$ $\mu \mathrm{M}$ of atRAL, a highly reactive compound that is cytotoxic to any cell it contacts $(29-32,49)$. Extensive studies in animals, including rats as well as WT and $A b c a 4^{---} R d h 8^{-/-}$double-KO mice that closely mimic many features of human retinal degeneration, showed that Ret- $\mathrm{NH}_{2}$ exhibits a protective effect against lightinduced damage by preventing buildup of atRAL and its condensation products $(7,33,50,51)$. But, Ret- $\mathrm{NH}_{2}$ is (a) used as a substrate by LRAT, (b) a less potent inhibitor of RPE65 than emixustat, and (c) metabolized to retinol in vivo $(7,26,33,35,39,50,52-54)$. First, Ret- $\mathrm{NH}_{2}$ is retained in the eye by the action of LRAT that produces its amidated precursor, and then the resulting retinyl amide is slowly hydrolyzed to evoke a long-lasting suppression of retinoid isomerase activity (35). Ret- $\mathrm{NH}_{2}$ acts as a substrate for LRAT that catalyzes the formation of retinylamides in vivo (35). Emixustat 
A

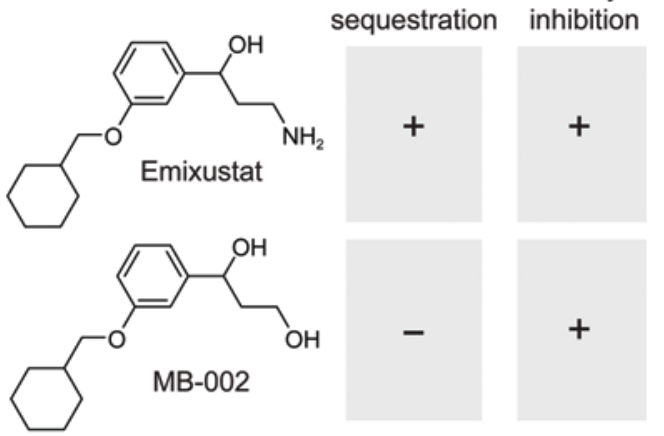

B

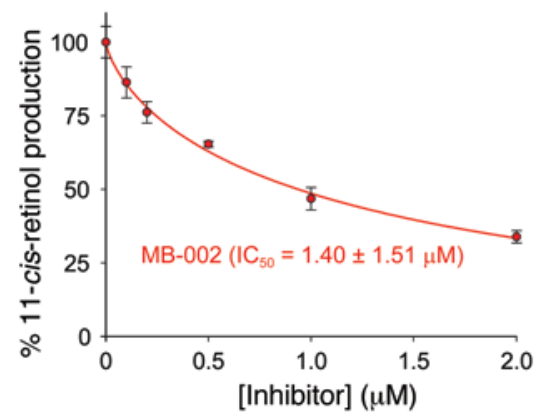

C

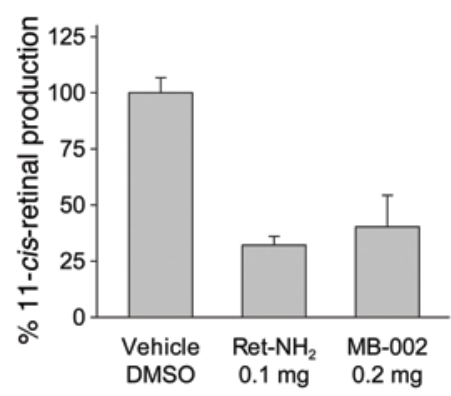

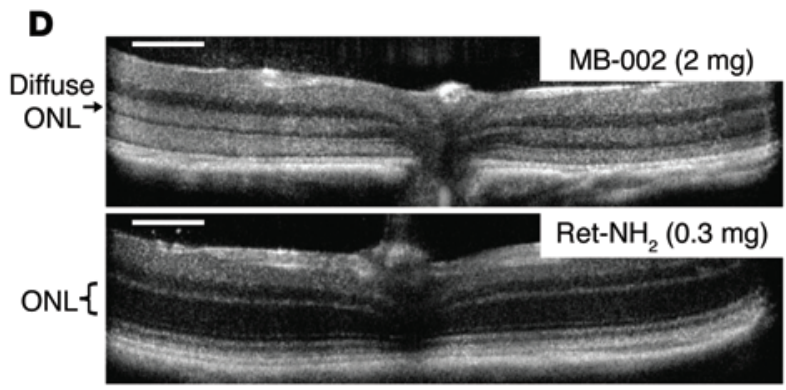

E

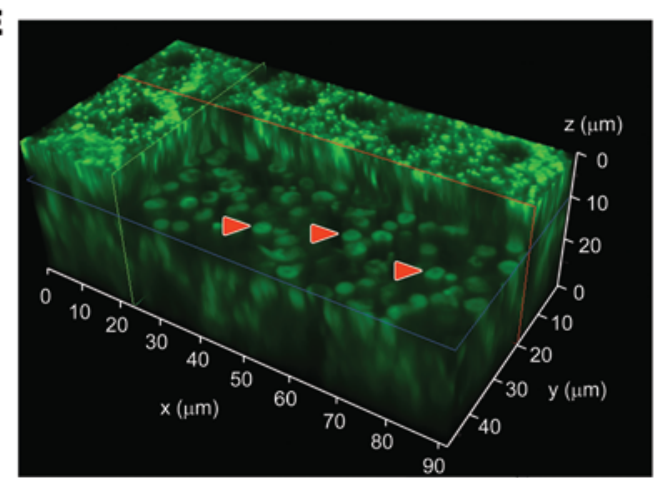

Figure 7. Effects of the emixustat derivative MB-002 on RPE65 activity and protection from light-induced retinopathy. (A) Chemical structures of emixustat and MB-002. (B) Inhibitory effect of MB-002 on retinoid isomerase activity in vitro. (C) Inhibitory effects of visual cycle modulators on 11-cis-retinal regeneration in [57BL6] mice. Post-bleaching recovery of ocular 11-cis-retinal was measured after a a 6-hour dark incubation period. (D) OCT images of retinae from pigmented $\mathrm{Abca} 4^{-/-} \mathrm{Rdh}^{-/-}$mice treated with MB-002 or retinylamine. The images, obtained 3 days after light exposure, show a lack of protective effects with MB-002. Scale bars: $100 \mu \mathrm{m}$. (E) Representative 3D TPM image of the retinal photoreceptor and RPE cell layers from an albino Abca4 ${ }^{-1}$ $R d h 8^{-/-}$mouse treated with MB-002 and exposed to intense light showing numerous swollen photoreceptor outer segments (red arrowheads). RPE cells are at the top of the section, and the photoreceptor cell layer section is shown at $10 \mu \mathrm{m}$ below the RPE. Mice were exposed to bright light 1 day after they were gavaged with either oil (control), Ret- $\mathrm{NH}_{2}$ ( $2 \mathrm{mg} /$ mouse), or MB-002 ( $2 \mathrm{mg} /$ mouse). TPM images of intact mouse eyes were obtained on day 2 after light exposure. Enlarged photoreceptors were counted in each group at a distance of 8 to $10 \mu \mathrm{m}$ from the RPE. The mean numbers ( \pm SD) of enlarged photoreceptors for each treatment group are as follows: $252 \pm 33$ for the oil-treated group; none in the no-light group; $5 \pm 5$ in Ret- $\mathrm{NH}_{2}$-treated animals; and $233 \pm 45$ in MB-002-treated animals. $n=4$.

also served as a moderate LRAT substrate and thus was absorbed by RPE cells as well (Figure 1C). Interestingly, although the efficacy of emixustat against retinal degeneration in $\mathrm{Abca}^{-/-} \mathrm{Rdh}^{-/-}$mice did not differ from that observed for Ret- $\mathrm{NH}_{2}$ and directly affected photoreceptor cells (Figure 5), the inhibitory effect on the retinoid cycle persisted much longer, suppressing production of the visual chromophore. Thus, the potential risk of adverse effects on photoreceptor health caused by prolonged inadequate regeneration of the visual chromophore appears to be much greater with emixustat, at least in mice $(24,26)$. We have also demonstrated that emixustat forms a surprisingly stable Schiff base with atRAL in vitro and that this complex can be trapped in vivo (Figure 6). Moreover, the protective effects of emixustat against light-induced retinal damage appear to be critically dependent on its ability to sequester atRAL, as evidenced by the failure of its diol derivative, MB-002, to protect against photoretinopathy in mice (Figure 7).

The disadvantage of this sequestration approach is the required high dose of the amino group-containing compound. Thus, a lead molecule must possess a high safety profile, achieve therapeutic inhibitory concentrations in the eye through reversible conjugation with atRAL, and only partially interfere with retinoid processing. If the conjugation between the amine and retinal is too avid, this could ultimately lead to toxicity due either to the formed product or to prolonged suppression of 11-cis-retinal production. Thus, only a partial slowing, but not a complete blockade, of visual chromophore regeneration, together with retinal sequestration activity, appears to be a viable therapeutic strategy for the prevention of many retinal degenerative diseases.

\section{Methods}

Synthetic chemistry. Synthesis of enantiopure emixustat and MB-002 was carried out as described in the supplemental material (Supplemental Figures 3-5).

TPM evaluation of the impact of retinoid cycle modulators on photoreceptor protection from bright light-induced damage. For these experiments, we used 4- to 5-week-old $A b c a 4^{-/-} R_{d h 8^{-/-}}$and BALB/c mice. After administering either $80 \mathrm{mg} / \mathrm{kg}$ Ret- $\mathrm{NH}_{2}, 80 \mathrm{mg} / \mathrm{kg}$ QEA-B001- $\mathrm{NH}_{2}, 40 \mathrm{mg} / \mathrm{kg}$ emixustat (racemic), $80 \mathrm{mg} / \mathrm{kg} \mathrm{MB}-002$, or 100 $\mu \mathrm{l}$ soybean oil with less than $10 \%$ (v/v) DMSO (control group), mice were placed in a dark room for 12 to 16 hours. Next, $\mathrm{Abca4}^{-/-} \mathrm{Rdh}^{-/-}$ 


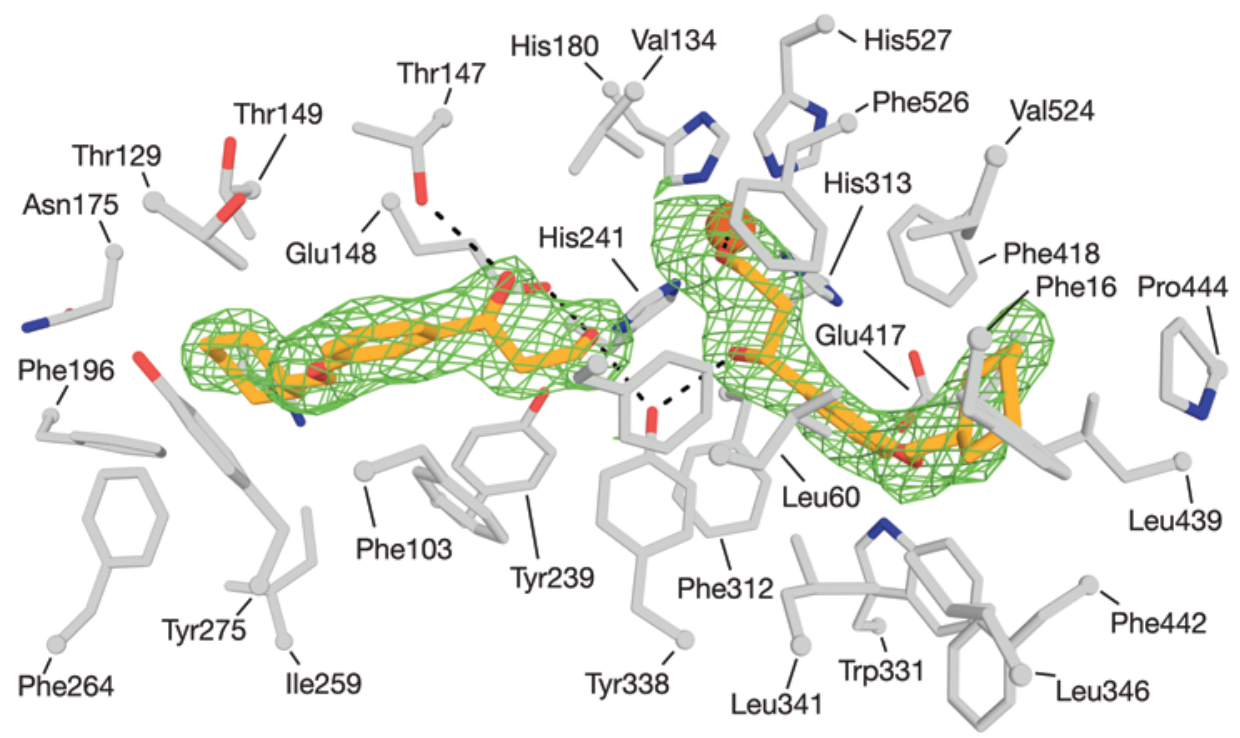

Figure 8. Crystal structure of RPE65 in complex with MB-002. The bound MB-002 molecules are shown as orange sticks. The $\sigma \mathrm{A}$-weighted Fo-Fc omit electron density is shown as green mesh contoured at $3 \sigma$. Residues within $4.5 \AA$ of the bound ligands are shown as gray sticks. mice were exposed to bright light at 10,000 lux for 1 hour, and $\mathrm{BALB} / \mathrm{c}$ mice were exposed to 20,000 lux for 2 hours as described below. These mice and a control group treated with soybean oil but not exposed to bright light were then kept in the dark for 2 days. On day 2 after light exposure, TPM was performed on intact eyes to evaluate the impact of the treatments on photoreceptors. A Leica TCS SP5 with Vision-S (Coherent) was used as described previously (31). All images were obtained with $730 \mathrm{~nm}$ excitation, $5 \mathrm{~mW}$ laser power, and the same settings of the detector and scanning parameters. Raw TPM images were used to quantify the impact of treatment. Enlarged photoreceptors were counted in the central/superior portion of the retina, 9-12 $\mu \mathrm{m}$ beneath the RPE, in an image frame corresponding to roughly $92 \times 92 \mu \mathrm{m}$. 3D sections were reconstructed from the series of $z$-stack images using Leica LAS-AF offline analysis software, version 3.1.2, for Leica TCS SP8.

RPE microsomal preparations. Bovine RPE microsomes were isolated from RPE homogenates by differential centrifugation as previously described (55). The resulting microsomal precipitate was resuspended in $10 \mathrm{mM}$ Bis-Tris propane/ $\mathrm{HCl}$ buffer, $\mathrm{pH}$ 7.4, to achieve a total protein concentration of approximately $5 \mathrm{mg} / \mathrm{ml}$. Then, the mixture was placed into a quartz cuvette and irradiated for 6 minutes at $4^{\circ} \mathrm{C}$ with a ChromatoUVE transilluminator (model TM-15; UVP) to eliminate residual retinoids. After irradiation, DTT was added to the RPE microsomal mixture to achieve a final concentration of $5 \mathrm{mM}$.

RPE65 crystallization and structure determination. Bovine RPE microsomes were isolated as previously reported (56). Proteins were extracted from the microsomes with $\mathrm{C}_{8} \mathrm{E}_{6}$, and RPE65 was purified from the sample by ion-exchange chromatography (56). Racemic, $(R)$ or $(S)$-emixustat, or MB-002, delivered in N,N-dimethylformamide (DMF), was added to the microsomal suspensions to achieve a final concentration of 1 to $2 \mathrm{mM}$ during the detergent extraction portion of the purification procedure. RPE65-containing fractions from ionexchange chromatography were pooled and concentrated to 10 to 15 $\mathrm{mg}$ protein/ml. RPE65 purity was judged to be greater than $90 \%$ on the basis of Coomassie-stained SDS-PAGE gels. Prior to crystallization trials, additional emixustat or MB-002 was added to the concentrated samples to achieve final concentrations of 1 and $5 \mathrm{mM}$, respectively.
Crystallization trials were performed by the hanging-drop vapor diffusion method using the Wizard Cryo I sparse matrix screen (Rigaku). Crystals were typically observed under several conditions. Well-diffracting, untwinned crystals were obtained under conditions no. 11 [35\% v/v MPD, $200 \mathrm{mM}\left(\mathrm{NH}_{4}\right)_{2} \mathrm{SO}_{4}$ and $100 \mathrm{mM}$ Tris- $\mathrm{HCl}, \mathrm{pH}$ 8.5]; no. 26 (40\% v/v PEG 300, $200 \mathrm{mM} \mathrm{NaCl}$ and $100 \mathrm{mM}$ CHES$\mathrm{NaOH}, \mathrm{pH} 9.5)$; and no. 15 (50\% PEG 400, 100 mM CHES, pH 9.5, and $200 \mathrm{mM} \mathrm{NaCl}$ ). These crystals grew to a final size of approximately $50 \times 50 \times 200 \mu \mathrm{m}$ within 1 week. Crystals were harvested with microloops (MiTeGen) and flash cooled in liquid nitrogen prior to x-ray exposure. No cryoprotective agents other than those present in the mother liquor were needed to suppress ice formation.

Diffraction data sets were collected on the NE-CAT ID-C and ID-E beamlines of the Advanced Photon Source (APS). Diffraction data were indexed, integrated, and scaled with the XDS package (57). Scaled intensities were converted to amplitudes by the French-Wilson procedure (58) as implemented in XDSCONV (57). Crystals of RPE65 obtained in the presence of racemic emixustat belonged to space group $P 6_{5} 22$ with 1 monomer in the asymmetric unit, whereas crystals containing $(R)$ - or $(S)$-emixustat or MB-002 belonged to space group $P 6_{5}$ with 2 monomers in the asymmetric unit. The absence of twinning was determined with phenix.xtraige (59).

Crystals were isomorphous to previously determined RPE65 crystal structures (Protein Data Bank [PDB] accession codes 4F3A and 3FSN, respectively), which enabled structure solution by rigid body refinement. Bound emixustat and palmitate ligands were clearly visible in the initial electron density maps obtained from crystals grown in the presence of emixustat (38). Electron density maps from MB-002 crystal were consistent with 2 bound MB-002 molecules in the active site. The MB-002 molecule occupying the emixustat binding site was found in the $(S)$ configuration, whereas electron density for the MB-002 in the palmitate binding site was consistent with the $(R)$-isomer. Adjustments to the protein structure and addition of water molecules were performed with Coot software (60). Restrained refinement was carried out using the REFMAC program (61). Following several cycles of manual model adjustments and computational refinement, the ligands were placed into the residual active site electron densities. 

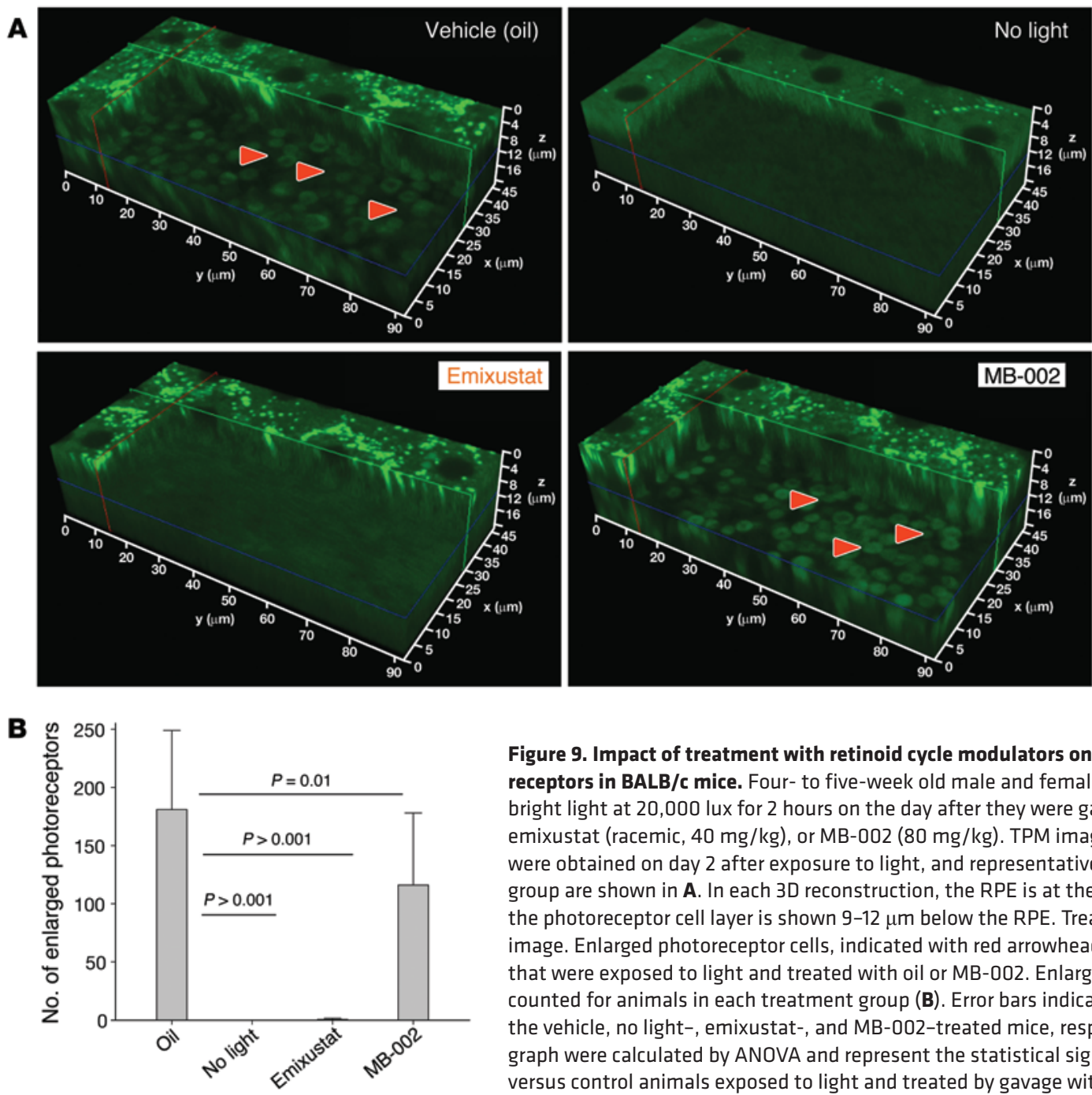

Figure 9. Impact of treatment with retinoid cycle modulators on the quantity of enlarged photoreceptors in BALB/c mice. Four- to five-week old male and female BALB/c mice were exposed to bright light at 20,000 lux for 2 hours on the day after they were gavaged with oil (control, $100 \mu \mathrm{l}$ ), emixustat (racemic, $40 \mathrm{mg} / \mathrm{kg}$ ), or MB-002 ( $80 \mathrm{mg} / \mathrm{kg}$ ). TPM images of intact BALB/c mouse eyes were obtained on day 2 after exposure to light, and representative 3D reconstructions for each group are shown in $\mathbf{A}$. In each $3 \mathrm{D}$ reconstruction, the RPE is at the top, and the section through the photoreceptor cell layer is shown 9-12 $\mu \mathrm{m}$ below the RPE. Treatments are indicated in each image. Enlarged photoreceptor cells, indicated with red arrowheads, were noted only in animals that were exposed to light and treated with oil or MB-002. Enlarged photoreceptor cells were counted for animals in each treatment group (B). Error bars indicate SDs; $n=8,5,8$, and 10 for the vehicle, no light-, emixustat-, and MB-002-treated mice, respectively. $P$ values shown in the graph were calculated by ANOVA and represent the statistical significance for each treated group versus control animals exposed to light and treated by gavage with oil.

Ligand models and geometric restraints were created with the Grade WebServer (Global Phasing). In the $P 6_{5} 22$ crystal form, residues $1-3,111-122,198-200$, and 267-271 were poorly resolved and thus excluded from the final model. Residues 110-126 and 197-201 were excluded from both chains in the $P 6_{5}$ crystal form for the same reason. Notably, the $N$ terminus, which was poorly ordered in all previously reported RPE65 structures, was well resolved in this $P 6_{5}$ crystal form. $\mathrm{N}$-terminal acetylation was evident in the electron density maps. The structures were validated with MolProbity (62), EDSTATS (63) from the CCP4 suite (64), and the wwPDB structure validation server (65). Final crystallographic statistics are shown in Table 1.

Coordinates and structure factor amplitudes have been deposited in the PDB (PDB accession codes 4RYX, 4RYY, 4RYZ, and 4ZHK) for the racemic emixustat, $(R)$-emixustat, $(S)$-emixustat, and MB-002 structures, respectively.

Retinoid isomerase activity assays. Synthesized primary amine $(2 \mu \mathrm{l}$ in DMF, with the final concentration ranging between 0.1 and $10 \mu \mathrm{M}$ ) was added to $10 \mathrm{mM}$ Bis-Tris propane/ $\mathrm{HCl}$ buffer, $\mathrm{pH}$ 7.4, containing $150 \mu \mathrm{g}$ RPE microsomes, $1 \%$ BSA, $1 \mathrm{mM}$ disodium pyrophosphate, and $20 \mu \mathrm{M}$ aporetinaldehyde-binding protein 1 (CRALBP). The resulting mixture was preincubated at room temperature for 5 minutes. Then, all-trans-retinol $(1 \mu \mathrm{l}$ in DMF, at a final concentration of $20 \mu \mathrm{M})$ was added. The resulting mixture was incubated at $37^{\circ} \mathrm{C}$ for 1 hour. The reaction was quenched by adding $300 \mu \mathrm{l}$ methanol, and products were extracted with $300 \mu$ l hexanes. Production of 11-cis-retinol was quantified by normal-phase HPLC with $10 \%$ (v/v) EtOAc in hexanes as the eluent at a flow rate of $1.4 \mathrm{ml} /$ minute. Retinoids were detected by monitoring absorbance at $325 \mathrm{~nm}$ and quantified based on a standard curve representing the relationship between the amount of synthetic 11-cis-retinol standard and the area under the corresponding chromatographic peak.

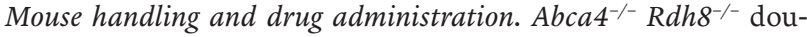
ble-KO mice on a C57BL/6J background were generated as previously described $(28,66)$. Male and female WT mice with C57BL/6J or BALB/cJ (BALB/c) backgrounds were obtained from The Jackson Laboratory. Abca4 $4^{-/-} \mathrm{Rdh}^{-/-}$mice used in this study were homozygous for the Leu 450 allele of Rpe 65 as determined by a genotyping protocol published previously (67) and free of Crb1/rd8 (68) and $\mathrm{rd} / \mathrm{rd}$ (69) mutations. Animals were housed in the animal facility at the Case Western Reserve University School of Medicine, where they were fed standard chow (LabDiet 5053) and maintained under a 12-hour light ( 300 lux) and 12-hour dark cycle. Mice were dark 
adapted overnight prior to experiments. A cohort of $\mathrm{Rdh}^{-/-} \mathrm{Abca4^{-/ }}$ and $\mathrm{BALB} / \mathrm{CJ}$ mice was used when they reached the age of 1 to 2 months, and both sexes were used evenly. All tested primary amines and visual cycle modulators were suspended in $100 \mu$ l soybean oil with less than $10 \%$ (v/v) DMSO and were administered by oral gavage with a 22-gauge feeding needle. Experimental manipulations in the dark were done under dim red light transmitted through a Kodak No. 1 Safelight Filter (transmittance $>560 \mathrm{~nm}$ ).

Induction of acute retinal degeneration in $\mathrm{Abca}^{-/-} \mathrm{Rdh}^{-/-}$mice. After dark adaptation for 24 hours, 4 -week-old male or female $\mathrm{Abca}^{-/-}$ $R d h 8^{-/-}$mice with pupils dilated by $1 \%$ tropicamide were exposed to fluorescent light (10,000 lux; $150 \mathrm{~W}$ spiral lamp; Commercial Electric) for 30 minutes in a white paper bucket (Papersmith) and then kept in the dark for an additional 3 days. The development of retinal degeneration was then examined by ultra-high-resolution spectral domain OCT (SD-OCT; Bioptigen) and ERG as previously described (10, 70).

Analysis of emixustat amides in mouse eyes. Emixustat $(8 \mathrm{mg} / \mathrm{kg})$ was administrated by oral gavage to 4 -week-old $\mathrm{Abca} 4^{-/-} \mathrm{Rdh8^{-/- }}$ mice, which were then kept in the dark for 24 hours. Then, mice were euthanized, and their eyes were homogenized in $1 \mathrm{ml}$ of $10 \mathrm{mM}$ sodium phosphate buffer, $\mathrm{pH} 7.4$, containing $50 \%$ methanol (v/v). The resulting mixture was extracted with $4 \mathrm{ml}$ EtOAc. Extracts were dried in vacuo and reconstituted in $300 \mu \mathrm{l}$ ethanol. The solution $(100 \mu \mathrm{l})$ was analyzed by liquid chromatography-electrospray ionization-MS/ MS (LC-ESI-MS/MS) (4.6 mm × $150 \mathrm{~mm}$; Agilent ZORBAX Eclipse $\mathrm{XDB}-\mathrm{C} 18)$ with a gradient of acetonitrile in water $(0 \%-100 \%$ in 30 minutes) as eluent at a flow rate of $0.5 \mathrm{ml} /$ minute.

Visual chromophore recovery assay. After bright light exposure that resulted in photoactivation of $95 \%$ of the rhodopsin, mice were kept in the dark for 2 hours to 7 days. Then, the animals were euthanized, and their eyes were collected and homogenized in $10 \mathrm{mM}$ sodium phosphate buffer, $\mathrm{pH} 7.4$, containing 50\% methanol (v/v) and $40 \mathrm{mM}$ hydroxylamine. The resulting mixture was extracted with $4 \mathrm{ml}$ of hexanes. Extracts were dried in vacuo, reconstituted in $300 \mu$ lof hexanes, and $100 \mu \mathrm{l}$ of the extract was injected into an HPLC system for analysis with $10 \%(\mathrm{v} / \mathrm{v})$ EtOAc in hexanes as the eluent.

MS-based detection of atRAL conjugates with primary amines. MS was performed with an LXQ linear ion trap mass spectrometer (Thermo Scientific) coupled to an Agilent 1100 HPLC system (Agilent Technologies). Separation of retinoids was achieved on a reversephase C18 Phenomenex HPLC Column $(250 \times 4.60 \mathrm{~mm} ; 5 \mu \mathrm{m})$ in a linear gradient of acetonitrile in water of $50 \%$ to $100 \%$ within $30 \mathrm{~min}$ utes at a flow rate of $1.5 \mathrm{ml} / \mathrm{minute}$. All solvents contained $0.1 \%$ formic acid (v/v). The HPLC effluent was directed into the mass spectrome- ter via an atmospheric pressure chemical ionization (APCI) interface operated in the positive ionization mode. Parameters of the ionization and detection were tuned with synthetic standards to achieve the highest possible sensitivity. In eye extracts, the emixustat-retinylidene Schiff base was detected in a selected reaction monitoring (SRM) mode using $m / z 530.5 \rightarrow 438.5$ transitions.

Statistics. Data representing the means \pm SD for the results of at least 3 independent experiments were compared by 1-way ANOVA. Differences with $P$ values of less than 0.05 were considered statistically significant.

Study approval. All animal procedures and experiments were approved by the Case Western Reserve University Animal Care Committee and conformed to recommendations of the American Veterinary Medical Association Panel on Euthanasia and the Association of Research for Vision and Ophthalmology.

\section{Acknowledgments}

We thank Leslie T. Webster Jr. and members of the Palczewski laboratory for helpful comments on this manuscript. This work was supported by funding from the NIH (EYO09339 and EY021126, to K. Palczewski; CA157735, to G.P. Tochtrop; EY025451 and AG043645, to G. Palczewska; and EY023948, to M. Golczak); the Department of Veterans Affairs (BX002683, to P.D. Kiser); the Foundation Fighting Blindness (to K. Palczewski); and the National Science Foundation (MCB-084480, to G.P. Tochtrop). We thank the staff at the APS NE-CAT beamlines for assistance with diffraction data collection. This work is based on research conducted at the APS on the Northeastern Collaborative Access Team beamlines, which are supported by a grant from the National Institute of General Medical Sciences (P41 GM103403) of the NIH. This research also used resources of the APS, a U.S. Department of Energy (DOE) Office of Science User Facility operated for the DOE Office of Science by Argonne National Laboratory under contract DE-AC02-06CH11357. We also thank Yinghua Chen of the Protein Expression Purification and Crystallography Core facility (PEPCC), Department of Physiology and Biophysics, Case Western Reserve University for assistance with circular dichroism (CD) data collection. K. Palczewski is a John H. Hord Professor of Pharmacology.

Address correspondence to: Krzysztof Palczewski, Department of Pharmacology, School of Medicine, Case Western Reserve University, 10900 Euclid Ave., Cleveland, Ohio 44106, USA. Phone: 216.368.4631; E-mail:kxp65@case.edu.
1. Batten ML, et al. Lecithin-retinol acyltransferase is essential for accumulation of all-trans-retinyl esters in the eye and in the liver. J Biol Chem. 2004;279(11):10422-10432.

2. Redmond TM, Poliakov E, Yu S, Tsai JY, Lu Z, Gentleman S. Mutation of key residues of RPE65 abolishes its enzymatic role as isomerohydrolase in the visual cycle. Proc Natl Acad Sci U S A. 2005;102(38):13658-13663.

3. Palczewski K, et al. Crystal structure of rhodopsin: a G protein-coupled receptor. Science. 2000;289(5480):739-745.

4. Palczewski K. G protein-coupled receptor rhodopsin. Annu Rev Biochem. 2006;75:743-767.

5. Kiser PD, Golczak M, Palczewski K. Chemistry of the retinoid (visual) cycle. Chem Rev. 2014;114(1):194-232.

6. Travis GH, Golczak M, Moise AR, Palczewski K. Diseases caused by defects in the visual cycle: retinoids as potential therapeutic agents. Annu Rev Pharmacol Toxicol. 2007;47:469-512.

7. Maeda A, Maeda T, Golczak M, Palczewski K. Retinopathy in mice induced by disrupted all-trans-retinal clearance. J Biol Chem. 2008;283(39):26684-26693.

8. Tsybovsky Y, Molday RS, Palczewski K. The
ATP-binding cassette transporter ABCA4: structural and functional properties and role in retinal disease. Adv Exp Med Biol. 2010;703:105-125.

9. Azarian SM, Megarity CF, Weng J, Horvath DH, Travis GH. The human photoreceptor rim protein gene (ABCR): genomic structure and primer set information for mutation analysis. Hum Genet. 1998;102(6):699-705.

10. Maeda T, et al. Evaluation of potential therapies for a mouse model of human age-related macular degeneration caused by delayed all-trans-retinal clearance. Invest Ophthalmol Vis Sci. 2009;50(10):4917-4925. 
11. Mata NL, Weng J, Travis GH. Biosynthesis of a major lipofuscin fluorophore in mice and humans with $\mathrm{ABCR}$-mediated retinal and macular degeneration. Proc Natl Acad Sci U S A. 2000;97(13):7154-7159.

12. Parish CA, Hashimoto M, Nakanishi K, Dillon J, Sparrow J. Isolation and one-step preparation of $\mathrm{A} 2 \mathrm{E}$ and iso-A2E, fluorophores from human retinal pigment epithelium. Proc Natl Acad Sci U S A. 1998;95(25):14609-14613.

13. Fishkin NE, Sparrow JR, Allikmets R, Nakanishi $\mathrm{K}$. Isolation and characterization of a retinal pigment epithelial cell fluorophore: an all-transretinal dimer conjugate. Proc Natl Acad Sci U S A. 2005;102(20):7091-7096.

14. Sparrow JR, et al. A2E, a byproduct of the visual cycle. Vision Res. 2003;43(28):2983-2990.

15. Sparrow JR, Nakanishi K, Parish CA. The lipofuscin fluorophore A2E mediates blue light-induced damage to retinal pigmented epithelial cells. Invest Ophthalmol Vis Sci. 2000;41(7):1981-1989.

16. Ach T, et al. Quantitative autofluorescence and cell density maps of the human retinal pigment epithelium. Invest Ophthalmol Vis Sci. 2014;55(8):4832-4841.

17. Ablonczy Z, et al. Lack of correlation between the spatial distribution of $\mathrm{A} 2 \mathrm{E}$ and lipofuscin fluorescence in the human retinal pigment epithelium. Invest Ophthalmol Vis Sci. 2013;54(8):5535-5542.

18. Sieving PA, et al. Inhibition of the visual cycle in vivo by 13-cis retinoic acid protects from light damage and provides a mechanism for night blindness in isotretinoin therapy. Proc Natl Acad Sci U S A. 2001;98(4):1835-1840.

19. Radu RA, Mata NL, Nusinowitz S, Liu X, Sieving PA, Travis GH. Treatment with isotretinoin inhibits lipofuscin accumulation in a mouse model of recessive Stargardt's macular degeneration. Proc Natl Acad Sci U S A. 2003;100(8):4742-4747.

20. Radu RA, et al. Reductions in serum vitamin $A$ arrest accumulation of toxic retinal fluorophores: a potential therapy for treatment of lipofuscin-based retinal diseases. Invest Ophthalmol Vis Sci. 2005;46(12):4393-4401.

21. Kubota R, Al-Fayoumi S, Mallikaarjun S, Patil S, Bavik C, Chandler JW. Phase 1, dose-ranging study of emixustat hydrochloride (ACU-4429), a novel visual cycle modulator, in healthy volunteers. Retina. 2014;34(3):603-609.

22. Kubota R, Boman NL, David R, Mallikaarjun S, Patil S, Birch D. Safety and effect on rod function of ACU-4429, a novel small-molecule visual cycle modulator. Retina. 2012;32(1):183-188.

23. Han M, Lou J, Nakanishi K, Sakmar TP, Smith SO. Partial agonist activity of 11-cis-retinal in rhodopsin mutants. J Biol Chem. 1997;272(37):23081-23085.

24. Woodruff ML, Wang Z, Chung HY, Redmond TM, Fain GL, Lem J. Spontaneous activity of opsin apoprotein is a cause of Leber congenital amaurosis. Nat Genet. 2003;35(2):158-164.

25. Cideciyan AV. Leber congenital amaurosis due to RPE65 mutations and its treatment with gene therapy. Prog Retin Eye Res. 2010;29(5):398-427.

26. Maeda T, et al. Loss of cone photoreceptors caused by chromophore depletion is partially prevented by the artificial chromophore prodrug, 9-cis-retinyl acetate. Hum Mol Genet . 2009;18(12):2277-2287.
27. Nickell S, Park PS, Baumeister W, Palczewski $\mathrm{K}$. Three-dimensional architecture of murine rod outer segments determined by cryoelectron tomography. J Cell Biol. 2007;177(5):917-925.

28. Maeda A, et al. Involvement of all-trans-retinal in acute light-induced retinopathy of mice. J Biol Chem. 2009;284(22):15173-15183.

29. Chen Y, et al. Mechanism of all-trans-retinal toxicity with implications for stargardt disease and age-related macular degeneration. J Biol Chem. 2012;287(7):5059-5069.

30. Maeda T, Golczak M, Maeda A. Retinal photodamage mediated by all-trans-retinal. Photochem Photobiol. 2012;88(6):1309-1319.

31. Maeda A, et al. Two-photon microscopy reveals early rod photoreceptor cell damage in lightexposed mutant mice. Proc Natl Acad Sci U S A. 2014;111(14):E1428-E1437.

32. Maeda A, et al. Primary amines protect against retinal degeneration in mouse models of retinopathies. Nat Chem Biol. 2012;8(2):170-178.

33. Golczak M, Kuksa V, Maeda T, Moise AR, Palczewski K. Positively charged retinoids are potent and selective inhibitors of the trans-cis isomerization in the retinoid (visual) cycle. Proc Natl Acad Sci U S A. 2005;102(23):8162-8167.

34. Zhang J, et al. Expansion of first-in-class drug candidates that sequester toxic all-trans-retinal and prevent light-induced retinal degeneration. Mol Pharmacol. 2015;87(3):477-491.

35. Golczak M, Imanishi Y, Kuksa V, Maeda T, Kubota R, Palczewski K. Lecithin:retinol acyltransferase is responsible for amidation of retinylamine, a potent inhibitor of the retinoid cycle. J Biol Chem. 2005;280(51):42263-42273.

36. Moise AR, Noy N, Palczewski K, Blaner WS. Delivery of retinoid-based therapies to target tissues. Biochemistry. 2007;46(15):4449-4458.

37. Palczewski K. Retinoids for treatment of retinal diseases. Trends Pharmacol Sci. 2010;31(6):284-295.

38. Kiser PD, et al. Catalytic mechanism of a retinoid isomerase essential for vertebrate vision [published online ahead of print April 20, 2015]. Nat Chem Biol. doi:10.1038/nchembio.1799.

39. Maeda A, et al. Effects of potent inhibitors of the retinoid cycle on visual function and photoreceptor protection from light damage in mice. $\mathrm{Mol}$ Pharmacol. 2006;70(4):1220-1229.

40. Wu X, et al. Synthesis and evaluation of a nanoglobular dendrimer 5-aminosalicylic Acid conjugate with a hydrolyzable schiff base spacer for treating retinal degeneration. ACS Nano. 2014;8(1):153-161.

41. Wenzel A, Reme CE, Williams TP, Hafezi F, Grimm C. The Rpe65 Leu450Met variation increases retinal resistance against light-induced degeneration by slowing rhodopsin regeneration. JNeurosci. 2001;21(1):53-58.

42. Moore JH, Hwa J. Editorial: pharmacogenetics and molecular medicine: "so close and yet so far." Curr Mol Med. 2014;14(7):803-804.

43. Damico FM, Gasparin F, Scolari MR, Pedral LS, Takahashi BS. New approaches and potential treatments for dry age-related macular degeneration. Arq Bras Oftalmol. 2012;75(1):71-76.

44. Evans JB, Syed BA. New hope for dry AMD? Nat Rev Drug Discov. 2013;12(7):501-502.

45. Fan J, Crouch RK, Kono M. Light prevents exoge- nous 11-cis retinal from maintaining cone photoreceptors in chromophore-deficient mice. Invest Ophthalmol Vis Sci. 2011;52(5):2412-2416.

46. Fan J, Rohrer B, Frederick JM, Baehr W, Crouch RK. Rpe65-/- and Lrat-/- mice: comparable models of leber congenital amaurosis. Invest Ophthalmol Vis Sci. 2008;49(6):2384-2389.

47. Rohrer B, Lohr HR, Humphries P, Redmond TM, Seeliger MW, Crouch RK. Cone opsin mislocalization in Rpe65-/- mice: a defect that can be corrected by 11-cis retinal. Invest Ophthalmol Vis Sci. 2005;46(10):3876-3882.

48. Znoiko SL, Rohrer B, Lu K, Lohr HR, Crouch RK, Ma JX. Downregulation of cone-specific gene expression and degeneration of cone photoreceptors in the Rpe65-/- mouse at early ages. Invest Ophthalmol Vis Sci. 2005;46(4):1473-1479.

49. Palczewska G, et al. Noninvasive multiphoton fluorescence microscopy resolves retinol and retinal condensation products in mouse eyes. Nat Med. 2010;16(12):1444-1449.

50. Golczak M, et al. Metabolic basis of visual cycle inhibition by retinoid and nonretinoid compounds in the vertebrate retina. J Biol Chem. 2008;283(15):9543-9554.

51. Berkowitz BA, et al. Quantitative mapping of ion channel regulation by visual cycle activity in rodent photoreceptors in vivo. Invest Ophthalmol Vis Sci. 2009;50(4):1880-1885.

52. Maeda A, Maeda T, Imanishi Y, Golczak M, Moise AR, Palczewski K. Aberrant metabolites in mouse models of congenital blinding diseases: formation and storage of retinyl esters. Biochemistry. 2006;45(13):4210-4219.

53. Tu DC, et al. Inner retinal photoreception independent of the visual retinoid cycle. Proc Natl Acad Sci U S A. 2006;103(27):10426-10431.

54. Schonthaler HB, et al. Evidence for RPE65-independent vision in the cone-dominated zebrafish retina. Eur J Neurosci. 2007;26(7):1940-1949.

55. Stecher H, Palczewski K. Multienzyme analysis of visual cycle. Methods Enzymol. 2000;316:330-344.

56. Kiser PD, Golczak M, Lodowski DT, Chance MR, Palczewski K. Crystal structure of native RPE65, the retinoid isomerase of the visual cycle. Proc Natl Acad Sci USA. 2009;106(41):17325-17330.

57. Kabsch W. XDS. Acta Crystallogr D Biol Crystallogr. 2010;66(Pt 2):125-132.

58. French S, Wilson K. On the treatment of negative intensity observations. Acta Crystallogr A. 1978;34(4):517-525.

59. Zwart PH, Grosse-Kunstleve RW, Lebedev AA, Murshudov GN, Adams PD. Surprises and pitfalls arising from (pseudo)symmetry. Acta Crystallogr D Biol Crystallogr. 2008;64(Pt 1):99-107.

60. Emsley P, Lohkamp B, Scott WG, Cowtan K. Features and development of Coot. Acta Crystallogr D Biol Crystallogr. 2010;66(Pt 4):486-501.

61. Murshudov GN, et al. REFMAC5 for the refinement of macromolecular crystal structures. Acta Crystallogr D. 2011;67(Pt 4):355-367.

62. Chen VB, et al. MolProbity: all-atom structure validation for macromolecular crystallography. Acta Crystallogr D Biol Crystallogr. 2010;66(Pt 1):12-21.

63. Tickle IJ. Statistical quality indicators for electron-density maps. Acta Crystallogr D Biol Crystallogr. 2012;68(Pt 4):454-467.

64. Winn MD, et al. Overview of the CCP4 suite and 
current developments. Acta Crystallogr D Biol Crystallogr. 2011;67(Pt 4):235-242.

65 . Read RJ, et al. A new generation of crystallographic validation tools for the protein data bank. Structure. 2011;19(10):1395-1412.

66. Chen Y, et al. Systems pharmacology identifies drug targets for Stargardt disease-associated retinal degeneration. JClin Invest. 2013;123(12):5119-5134.
67. Grimm C, et al. Constitutive overexpression of human erythropoietin protects the mouse retina against induced but not inherited retinal degeneration. J Neurosci. 2004;24(25):5651-5658.

68. Mattapallil MJ, et al. The Rd8 mutation of the $\mathrm{Crb} 1$ gene is present in vendor lines of $\mathrm{C} 57 \mathrm{BL} / 6 \mathrm{~N}$ mice and embryonic stem cells, and confounds ocular induced mutant phenotypes. Invest Oph- thalmol Vis Sci. 2012;53(6):2921-2927.

69. Pittler SJ, Baehr W. Identification of a nonsense mutation in the rod photoreceptor cGMP phosphodiesterase beta-subunit gene of the rd mouse. Proc Natl Acad Sci U S A. 1991;88(19):8322-8326.

70. Zhang N, et al. Autosomal recessive retinitis pigmentosa E150K opsin mice exhibit photoreceptor disorganization. JClin Invest. 2013;123(1):121-137. 